\title{
Molecular line emission in HH54: a coherent view from near to far infrared ${ }^{\star}$
}

\author{
T. Giannini ${ }^{1}$, C. $\mathrm{M}^{\mathrm{c}} \mathrm{Coey}^{2}$, B. Nisini ${ }^{1}$, S. Cabrit ${ }^{3}$, A. Caratti o Garatti ${ }^{4}$, L.Calzoletti $^{1,5}$, and D. R. Flower ${ }^{6}$ \\ 1 INAF-Osservatorio Astronomico di Roma, via Frascati 33, 00040 Monte Porzio Catone, Italy \\ e-mail: [giannini;nisini;calzol]@oa-roma.inaf.it \\ 2 Department of Physics, University of Waterloo 200, University Avenue W., Ontario, N2L 3G1, Canada \\ e-mail: cmccoey@astro.uwaterloo.ca \\ 3 LERMA, Observatoire de Paris, UMR 8112, 75014 Paris, France \\ e-mail: sylvie.cabrit@obspm.fr \\ 4 Thüringer Landessternwarte, Sternwarte 5, 07778 Tautenburg, Germany \\ e-mail: caratti@tls-tautenburg.de \\ 5 Università di Cagliari, via Università 40, 09124 Cagliari, Italy \\ ${ }^{6}$ Physics Department, Durham University, Durham, DH1 3LE, UK \\ e-mail: david.flower@durham.ac.uk
}

Received 2 March 2006 / Accepted 23 August 2006

\section{ABSTRACT}

\begin{abstract}
Aims. We present a detailed study of the infrared line emission (1-200 $\mu \mathrm{m})$ in the Herbig-Haro object HH54. Our database comprises: high- $(R \sim 9000)$ and low- $(R \sim 600)$ resolution spectroscopic data in the near-infrared band $(1-2.5 \mu \mathrm{m})$; mid-infrared spectrophotometric images $(5-12 \mu \mathrm{m})$; and, far-IR $(45-200 \mu \mathrm{m}, R \sim 200)$ spectra acquired with the ISO satellite. As a result, we provide the detection of and the absolute fluxes for more than 60 molecular features (mainly from $\mathrm{H}_{2}$ in the near- and mid-infrared and from $\mathrm{H}_{2} \mathrm{O}$, $\mathrm{CO}$ and $\mathrm{OH}$ in the far-infrared) and 23 ionic lines.

Methods. The $\mathrm{H}_{2}$ lines, coming from levels from $v=0$ to $v=4$ have been interpreted in the context of a state-of-the-art shock code, whose output parameters are adopted as input to a Large Velocity Gradient computation in order to interpret the FIR emission of CO, $\mathrm{H}_{2} \mathrm{O}$ and $\mathrm{OH}$

Results. The $\mathrm{H}_{2}$ emission can be interpreted as originating in either steady-state J-type shocks or in quasi-steady J-type shocks with magnetic precursor. However, our multi-species analysis shows that only a model of a J-type shock with magnetic precursor $\left(v_{\text {shock }}=\right.$ $18 \mathrm{~km} \mathrm{~s}^{-1}, n_{\mathrm{H}}=10^{4} \mathrm{~cm}^{-3}, B=100 \mu \mathrm{G}$, age $=400 \mathrm{yr}$ ) can account for both the observed $\mathrm{H}_{2}$ emission and the $\mathrm{CO}$ and $\mathrm{H}_{2} \mathrm{O}$ lines. Such a model predicts a $\mathrm{H}_{2} \mathrm{O}$ abundance of $\sim 7 \times 10^{-5}$, in agreement with estimations from other shock models of outflows associated with low mass protostars. We can exclude the possibility that the observed atomic lines arise in the same shock as the molecular lines, and give arguments in favour of the presence of a further high-velocity, fully dissociative shock component in the region. Finally, in view of the forthcoming spectroscopic facilities on board of the Herschel satellite, we provide predictions for $\mathrm{H}_{2} \mathrm{O}$ lines considered to be the most suitable for diagnostic purposes.
\end{abstract}

Key words. stars: circumstellar matter - infrared: ISM - ISM: Herbig-Haro objects - ISM: individual objects: HH54 -

ISM: jets and outflows

\section{Introduction}

It is now commonly accepted that the earliest stages of star formation are accompanied by powerful bipolar jets, which act to remove excess angular momentum from in-falling gas (e.g. Bachiller 1996). The jets are generally highly collimated and often terminate in a curve-shaped structure (bow-shock); however, knots of shocked emission, which may display a bow morphology, are also observed along the jet (e.g. Reipurth \& Bally 2001). The thermal energy associated with a shock is radiated away through the emission of lines from atomic, ionic and molecular species. Gas at temperatures of thousands of degrees Kelvin

* Based on observations collected at the European Southern Observatory (La Silla and Paranal), Chile (65.I-0150, 70.C-0138, 74.C-0235) and observations with ISO,pa an ESA project with instruments funded by ESA Members States (especially the PI countries: France, Germany, The Netherlands, and the UK) and with the participation of ISAS and NASA. cools principally through ro-vibrational lines of molecular hydrogen in the near-infrared, but also through forbidden transitions of abundant atomic and ionic species, such as [Fe II], [S II], $[\mathrm{CI}]$ and $[\mathrm{NI}]$. Colder gas components, at hundreds of degrees Kelvin, cool via mid- and far-infrared molecular lines, particularly rotational transitions of $\mathrm{H}_{2}$ (at $\left.\lambda \leq 28 \mu \mathrm{m}\right)$ and lines of other molecular species, such as $\mathrm{H}_{2} \mathrm{O}, \mathrm{CO}$ and $\mathrm{OH}$ (in the far-infrared). The relative contribution to the gas cooling depends strongly upon the physical conditions within the shock. In particular, the shock velocity and the conditions in the pre-shock gas (the degree of ionization, the strength of the magnetic field and the density) determine the structure of the shock wave (Draine 1980), which is usually classified as "jump" (J-type), "J-type with magnetic precursor" or "continuous" (C-type) (e.g., Hollenbach \& McKee 1989). In general, J-type shocks produce a narrower profile and a higher peak temperature than C-type shocks while J-type shocks with magnetic precursor can be considered to be intermediate. J-type shocks can heat the gas up to temperatures 
Table 1. Journal of observations.

\begin{tabular}{|c|c|c|c|c|c|c|c|}
\hline Telescope/Instrument & Date & \multicolumn{2}{|l|}{ Filter } & $\begin{array}{c}\text { FoV } \\
\left({ }^{\prime}\right) \times\left({ }^{\prime}\right)\end{array}$ & \multicolumn{2}{|c|}{$\begin{array}{l}\text { Pixel scale } \\
(" / p x l)\end{array}$} & $\begin{array}{c}\text { Exposure time } \\
\text { (s) }\end{array}$ \\
\hline NTT/SofI & 6 Jun. 1999 & \multicolumn{2}{|c|}{$\mathrm{H}_{2} 2.12 \mu \mathrm{m}$} & $4.9 \times 4.9$ & \multicolumn{2}{|c|}{0.29} & 300 \\
\hline ISO/ISOCAM & 20 Oct. 1997 & \multicolumn{2}{|c|}{ CVF 5.0-16.8 $\mu \mathrm{m}$} & $3 \times 3$ & \multicolumn{2}{|c|}{6} & 3600 \\
\hline \multicolumn{8}{|c|}{ Spectroscopy } \\
\hline Telescope/Instrument & Date & $\begin{array}{c}\lambda \\
(\mu \mathrm{m})\end{array}$ & Slit/c & iperture & $\begin{array}{l}\text { PA } \\
\left({ }^{\circ}\right)\end{array}$ & $\lambda / \Delta \lambda$ & $\begin{array}{l}\text { Exposure time } \\
\text { (s) }\end{array}$ \\
\hline VLT/ISAAC & 11 Jul. 2002 & $0.98-2.50$ & & I & 28 & 1600 & 1600 \\
\hline NTT/SofI & 6 Jun. 1999 & $1.53-2.52$ & & II & 140 & 600 & 2400 \\
\hline VLT/ISAAC & 31 Dec. 2004 & $2.06-2.18$ & & /III & $28 / 160$ & 8900 & 1800 \\
\hline ISO/LWS & 17 Feb. 1996 & $43-197$ & & $80^{\prime \prime}$ & _ & 200 & 2740 \\
\hline ISO/LWS -FP & 23 Sep. 1997 & $63.13-63.23$ & & $80^{\prime \prime}$ & - & 8500 & 6000 \\
\hline
\end{tabular}

of $10^{5} \mathrm{~K}$ and can travel at up to hundreds of $\mathrm{km} \mathrm{s}^{-1}$, while C-type shock velocities do not exceed $\approx 80 \mathrm{~km} \mathrm{~s}^{-1}$ (Le Bourlot et al. 2002; Flower et al. 2003). Classically, calculations of shock structure have been performed under the assumption of steady state. However, if this assumption is relaxed it has been shown that, in the presence of a sufficiently strong magnetic field, an initially J-type discontinuity may evolve in time into a C-type shock wave (Smith \& MacLow 1997; Chièze et al. 1998). The intermediary stage of the evolution is described by a (quasi-steady) J-type shock wave with magnetic precursor and can be considered as a J-type discontinuity introduced into a C-type shock profile at the point in the ion fluid flow time, $t_{\mathrm{i}}=\int 1 / v_{\mathrm{i}} \mathrm{d} z$, that may be identified with the age of the shock (Lesaffre et al. 2004a).

Although 2D and 3D hydrodynamical and MHD simulations of jet-driven molecular shocks have progressed enormously over the last years, including some time-dependent $\mathrm{H}_{2}$ chemistry, $\mathrm{H}$ ionization, and cooling (Downes \& Cabrit 2003; Smith \& Rosen 2003), the effect of ion-neutral decoupling in magnetic precursors and the resulting complex shock chemistry have so far only been simulated in 1D (Chieze et al. 1998; Lesaffre et al. 2004b). Lesaffre et al. (2004a) showed that truncated steadystate multi-fluid models provide a useful and efficient approximation to time-dependent simulations, while allowing a more detailed treatment of the chemistry and hence of the degree of ionization. We shall thus adopt this approach here.

Since different shock types give rise to very different excitation conditions, the interpretation of multiple species observed over a wide range in wavelength provides strong constraints for a shock model. Multi-wavelength studies have already proved useful: for example, Smith et al. (2003) employed an analysis of the near- to far-infrared molecular emission observed towards Cepheus E in order to determine both the shock type and geometry. The main limitation of the multi-wavelength/multi-species approach arises from the poor sensitivity and, more importantly, the poor spatial resolution achieved in the mid- and far-infrared by space-borne instrumentation, which often prevents the resolution of structures along jets and in bow-shocks. In the near future, however, we expect that these limitations will be overcome by the instruments aboard the Herschel satellite, whose performance in terms of sensitivity and of spatial and spectral resolution will be closer to those attainable with ground-based observations at shorter wavelengths.

The aims of the present work are threefold. Firstly, we wish to test the ability of a current shock model to self-consistently reproduce emission from several different species observed over a wide range of wavelengths. Secondly, we will investigate which combinations of these observations prove most useful in determining shock conditions, particularly shock type. Finally, from this analysis we intend to identify important diagnostic molecular lines, mainly of $\mathrm{H}_{2} \mathrm{O}$, falling in the wavelength range observable with Herschel. As a test case, we have applied our analysis to the Herbig-Haro object HH54. This is located at the northern edge of the nearby star-forming region ChaII $(d \approx$ 200 pc, Hughes \& Hartigan 1992). The object, consisting of a complex of several arcsecond-scale bright knots, was originally discovered by Schwartz (1977), then spectroscopically observed in the optical by Schwartz \& Dopita (1980) and Graham \& Hartigan (1988), who found a blue-shifted gas with a radial velocity that becomes increasingly bluer in a long "streamer" from the north to the south and that may be a jet responsible for the excitation of the HH54 complex. Knee (1992, hereafter K92) associates HH54 with a monopolar blue-shifted CO outflow, whose driving source remains unclear after a proper motion analysis of the infrared knots (Caratti o Garatti et al. 2006). Molecular hydrogen emission was first reported by Sandell et al. (1987), while the near-infrared spectrum was obtained by Gredel (1994, hereafter G94). In the mid-infrared, observations with ISO/CAM (Cabrit et al. 1999), ISO/SWS (Neufeld et al. 1998), and quite recently, with Spitzer/IRS (Neufeld et al. 2006) have shown the $\mathrm{H}_{2}$ ortho-to-para ratio to lie below the equilibrium value expected at the observed gas temperature. Far-infrared emission, detected with ISO/LWS has been reported by Nisini et al. (1996) and discussed by Liseau et al. (1996) in the framework of planar, steady-state C-shocks. Conversely, Wilgenbus et al. (2000), who compared the predictions of steady-state shock models to observations of pure rotational (Neufeld et al. 1998) and ro-vibrational (G94) $\mathrm{H}_{2}$ lines, concluded that no single planar C- or J-type shock is able to account for the observed emission in both pure rotational and ro-vibrational lines. In the present work, we will check whether or not this result can be confirmed on the basis of a large observational data-base.

The structure of the paper is the following: in Sect. 2 the observations used for our analysis are presented, while in Sect. 3 the adopted shock model is described and applied to the data. Our conclusions are then summarized in Sect. 4.

\section{Observations and results}

Our database consists of data collected with the ESO facilities and retrieved from the ISO archive ${ }^{1}$. The relevant information on the observational settings is summarized as a log in Table 1.

\footnotetext{
1 Available at http://www. iso.vilspa.esa.es/ida/ index.html
} 


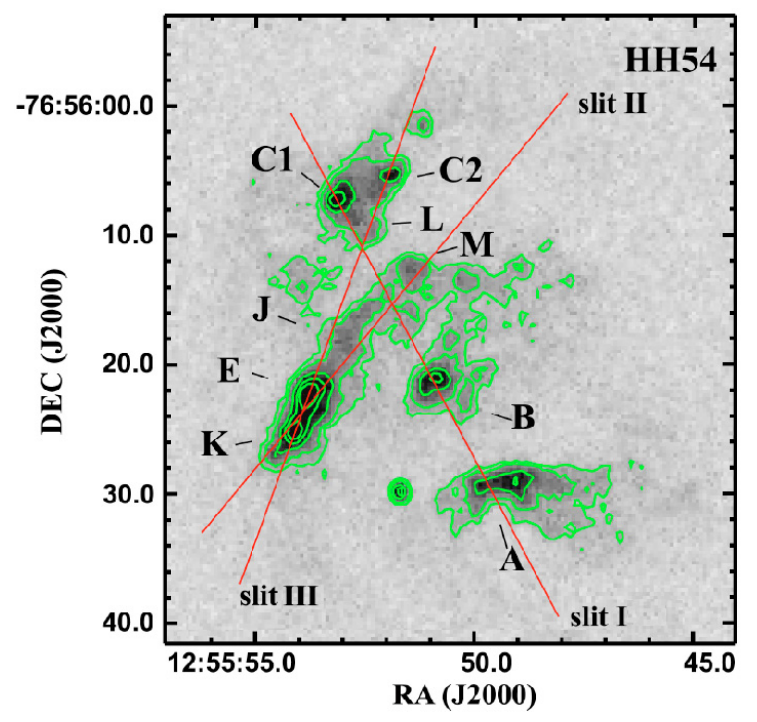

Fig. 1. $\mathrm{H}_{2} 2.12 \mu \mathrm{m}$ (continuum-subtracted) SofI image of HH54. The knots of emission are labelled according to the nomenclature of G94 and Sandell et al. (1987). The contours are 3, 5, 10, 15 and $20 \times$ the standard deviation to the mean background $\left(5 \times 10^{-15} \mathrm{erg} \mathrm{s}^{-1} \mathrm{~cm}^{-2} \operatorname{arcsec}^{-2}\right)$. The orientations of the slit used for spectroscopic observations are depicted.

\subsection{Ground-based observations}

\subsubsection{Imaging}

A narrow-band image in the $\mathrm{H}_{2}$ 1-0S(1) filter was obtained with SofI (Lidman et al. 2002) at the New Technology Telescope (NTT, La Silla, Chile). The observation was conducted by nodding and jittering the telescope around the pointed position $\left(\alpha(\mathrm{J} 2000)=12^{\mathrm{h}} 55^{\mathrm{m}} 50.3^{\mathrm{s}}, \delta(\mathrm{J} 2000)=-76^{\circ} 56^{\prime} 23^{\prime \prime}\right)$ in the usual ABB' A'mode. The raw data were reduced by using standard procedures for bad pixel removal, flat fielding and sky subtraction. Flux calibration was derived from the observation of a photometric standard star during the night. A continuum-free image was obtained by subtracting from the narrow band image an appropriately scaled $K_{\mathrm{s}}$ image, acquired just after the observation. The $\mathrm{H}_{2}$, continuum-subtracted image is shown in Fig. 1, where the knots of line emission are labelled according to the notation of G94 and Sandell et al. (1987). The HH54 complex appears to be composed of arcsecond-scale bright knots: those labelled A, $\mathrm{B}$ and $\mathrm{C}$ lie along the axis of the optical "streamer" extending south of HH54; for brevity, we will refer to this axis as the "jet axis" in the following. In addition, two further knots (E, K) plus (at least) three more diffuse regions are characterized by a bow-shaped morphology. Additional knots, roughly located along the axis and barely visible in the G94 image, have been detected (namely, J, L, M). The (1-0)S(1) line flux of the whole HH54 complex is $1.12 \times 10^{-12} \mathrm{erg} \mathrm{s}^{-1} \mathrm{~cm}^{-2}$, i.e. approximately a factor of two and five larger than the values measured by G94 and Sandell (1987), respectively. The brightness variability of the $\mathrm{H}_{2} 1-0 \mathrm{~S}(1)$ line on a time scale of about 10 years, pointed out by G94, is thus confirmed by the present observations. As suggested by Liseau et al. (1996), such variability indicates that HH54 is a non-equilibrium flow.

\subsubsection{Low-resolution spectroscopy}

Long-slit, near infrared spectra of the HH54 region were collected with the ESO facilities during two different runs. In 1999, we obtained the $1.55-2.50 \mu \mathrm{m}$ spectra of the knots along the brightest wing of the bow (knots E, K) with SofI, while the jet axis was targetted in 2002 with ISAAC (Cuby et al. 2004) at the Very Large Telescope (VLT, Paranal, Chile). Four grating settings ( $S Z, J, S H, S K$ filters) are required with ISAAC to cover the spectrum between $0.98-2.50 \mu \mathrm{m}$. The adopted slit orientations along with the knots encompassed by the slit are depicted in Fig. 1. The raw spectral images were flat-fielded, sky subtracted and corrected for the optical distortions along both the spatial and spectral directions. Telluric features were removed by dividing the extracted spectra by that of a blackbody-normalized telluric standard star (spectral type O9V), once corrected for its intrinsic spectral features. Wavelength calibration was derived from the lines of a xenon-argon lamp, while flux calibration was based on the photometry of the $\mathrm{H}_{2} 1-0 \mathrm{~S}(1)$ continuumsubtracted image, whose associated uncertainty is within $10 \%$. Ratios of lines falling in different portions of the ISAAC spectrum are affected by an uncertainty of up to $20 \%$, due to the different instrumental responses in the four segments. This error was estimated by comparing the fluxes of lines present in overlapping parts of the spectrum. Figure 2 and Table 2 show the ISAAC spectrum and the line fluxes detected in knot B, which is among the knots richest in lines. Clearly, regions at different degrees of dissociation and ionization are encompassed by the slit aperture: low excitation conditions are characterized by the presence of copious $\mathrm{H}_{2}$ ro-vibrational lines (with $v \leq 4$ and a maximum excitation energy of $\sim 25000 \mathrm{~K}$ ), while the presence of ionized gas at $T \approx 10000 \mathrm{~K}$ is evidenced from the detection of several forbidden lines from [Fe II], [C I ], [S II], [N I] along with hydrogen and helium recombination lines.

\subsection{3. $2.12 \mu \mathrm{m}$ medium-resolution spectroscopy}

Medium-resolution spectra of the $\mathrm{H}_{2} 2.1218 \mu \mathrm{m}$ line were obtained in January 2004 with ISAAC, along the directions depicted in Fig. 1 (slits I and III). The slit aperture is $0.3^{\prime \prime} \times 120^{\prime \prime}$, which gives a nominal resolution of about 8900 , i.e. $33.7 \mathrm{~km} \mathrm{~s}^{-1}$. The data were acquired and reduced with the same techniques outlined in the previous section. Wavelength calibration was performed using bright $\mathrm{OH}$ atmospheric lines (Rousselot et al. 2000), which provide an accuracy to within $3 \mathrm{~km} \mathrm{~s}^{-1}$. The instrumental profile in the dispersion direction, as measured from Gaussian fits to sky lines, was $\sim 31.7 \mathrm{~km} \mathrm{~s}^{-1}$. We give in Table 3 the velocity parameters for all the encompassed knots. The peak radial velocity $\left(v-v_{\text {cloud }}\right)$ was computed with respect to the ambient molecular cloud, for which a velocity of $2.0 \mathrm{~km} \mathrm{~s}^{-1}$ in the local standard of rest (LSR) has been adopted (K92). All the knots appear blue-shifted by about $10 \mathrm{~km} \mathrm{~s}^{-1}$, a circumstance which, although confirming the association of HH54 with a blue-shifted outflow, excludes the possibility that $\mathrm{H}_{2}$ emission originates in the same gas that gives rise to the optical spectrum, where $v_{\text {rad }}$ ranges from -20 to $-70 \mathrm{~km} \mathrm{~s}^{-1}$ (Graham \& Hartigan 1988). The observed FWHM is larger than the instrumental width by a few $\mathrm{km} \mathrm{s}^{-1}$ : the deconvolved values, which give an estimate of the velocity spread $(\Delta v)$, range between 13 and $20 \mathrm{~km} \mathrm{~s}^{-1}$. The poor spectral resolution, coupled with the low signal-to-noise ratio at the base of the line, prevents an accurate measurement of the intrinsic FWZI, which could provide some information about the speed of the bow-shock (Hartigan et al. 1987). An average velocity of $\approx 50 \mathrm{~km} \mathrm{~s}^{-1}$ along the jet axis, however, has been recently estimated from proper motion analysis by Caratti o Garatti et al. (2006). We can consider this value to be an upper limit to the shock velocity, given the possibility that the observed shocks travel in a medium already put into motion by previous ejection 

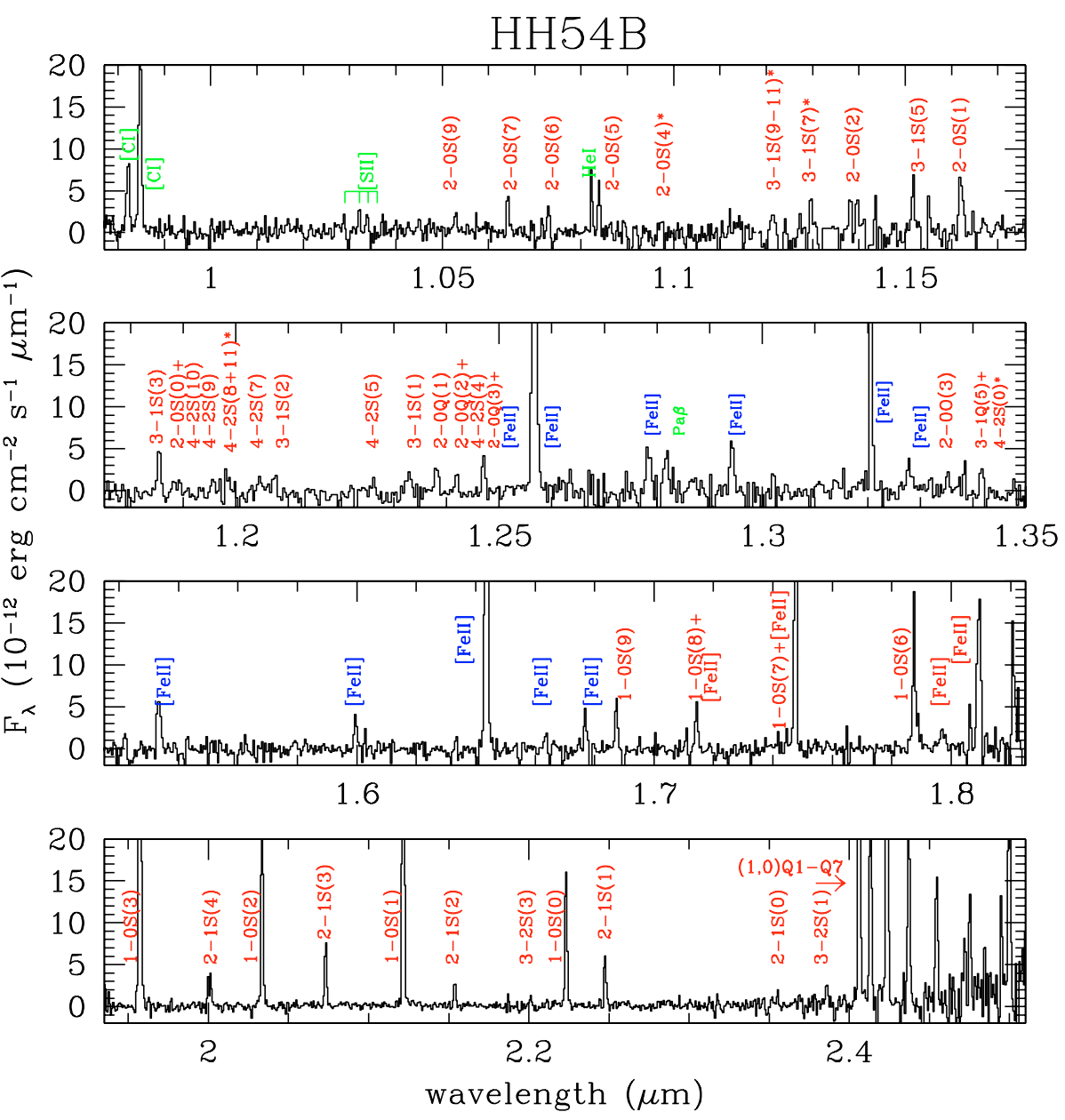

Fig. 2. 1.0-2.5 $\mu \mathrm{m}$ low resolution spectrum of HH54B. Lines marked with an asterisk are detected with signal-to-noise ratio less than 3.

events, a phenomenon that is frequently observed in the environments of protostellar outflow (e.g. Chrysostomou et al. 2000).

\subsection{Space-borne observations}

\subsubsection{Mid-infrared spectro-imaging}

Images of $\mathrm{HH} 54$ in the $\mathrm{H}_{2}$ pure rotational transitions from 0-0 $S(2)$ to $S(7)$ were obtained with the infrared camera ISOCAM (Cesarsky et al. 1996) aboard ISO, in the guaranteed time CAMFLOWS programme. Spectral scans were obtained with the Circular Variable Filter (CVF) over a wavelength range from 16.61 to $5.08 \mu \mathrm{m}$ with full spectral sampling, and a camera pixel size of $6^{\prime \prime}$ yielding a total field of view of about $3^{\prime}$ by $3^{\prime}$. The raw data files were retrived from the ISO archive and reprocessed locally with the CAM Interactive Analysis (CIA; Ott et al. 2001) software. The data reduction procedure consists of dark current and flat field correction and cosmic ray removal. Continuum was then removed under each $\mathrm{H}_{2}$ line by fitting a linear baseline, and the line flux was obtained by trapezoidal integration over 5 spectral elements, yielding individual images in each $\mathrm{H}_{2}$ line. A preliminary report of this dataset was presented by Cabrit et al. (1999). In the reduced images, shown in Fig. 3, the same bow-shape morphology delineated by the 1-0S(1) line is recognizable but the gas traced by the rotational lines appears smooth, rather than clumpy as in the $2.12 \mu \mathrm{m}$ image. This could be ascribed to the poor spatial resolution at mid-infrared wavelengths, but could also reflect a different mechanism at the origin of the excitation of the ro-vibrational and pure rotational lines. We will address this point further on in the paper (see Sect. 3.2).

The extent of the regions where emission is detected at over $3 \sigma$ above the sky level, increases from the highest frequency line $0-0 \mathrm{~S}(7)$ at $5.5 \mu \mathrm{m}$ to the $0-0 \mathrm{~S}(2)$ line at $12.3 \mu \mathrm{m}$, an effect which is due both to the loss of spatial resolution at increasing wavelength and to the progressively lower temperatures (from thousands to hundreds of Kelvin) probed by lines with lower excitation energy. As one of our aims is to obtain a meaningful comparison between far- and near-infrared lines, we have superposed the $0-0$ contours on the $2.12 \mu \mathrm{m}$ image, so as to identify the regions where the emission overlaps. The ISOCAM spatial resolution does not allow us to disentangle structures with an angular size of less than 6 arcsec, a dimension often larger than that of the near infrared knots. In the $5.5 \mu \mathrm{m}$ image, which better matches the morphology delineated at $2.12 \mu \mathrm{m}$, we are able to resolve four peaks roughly corresponding to the three knots from north to south (knots $\mathrm{C}$ to $\mathrm{A}$ ) and to the wing (knots $\mathrm{E}, \mathrm{K}$ ). Aiming to attribute a mid-infrared flux to each peak, we have defined four zones, each centred on the individual peak, and containing at least $10 \%$ of the peak emission; inside each zone the flux has been computed by using the task polyphot in the IRAF package ${ }^{2}$. The results are given in Table 4 , where each zone has been identified by indicating the corresponding knots

${ }^{2}$ IRAF (Image Reduction and Analysis Facility) is distributed by the National Optical Astronomy Observatories, which are operated by AURA, Inc., cooperative agreement with the National Science Foundation. 
Table 2. Near infrared line fluxes observed in knot B.

\begin{tabular}{|c|c|c|c|c|c|}
\hline Line id. & $\begin{array}{c}\lambda \\
(\mu \mathrm{m})\end{array}$ & $\begin{array}{c}F \pm \Delta F^{a} \\
\left(10^{-15} \mathrm{erg} \mathrm{cm}^{-2} \mathrm{~s}^{-1}\right)\end{array}$ & Line id. & $\begin{array}{c}\lambda \\
(\mu \mathrm{m})\end{array}$ & $\begin{array}{c}F \pm \Delta F^{a} \\
\left(10^{-15} \mathrm{erg} \mathrm{cm}^{-2} \mathrm{~s}^{-1}\right)\end{array}$ \\
\hline \multicolumn{6}{|c|}{$\mathrm{H}_{2}$ lines } \\
\hline $2-0 \mathrm{~S}(9)$ & 1.053 & $0.66 \pm 0.07$ & $3-1 \mathrm{Q}(7)$ & 1.368 & $0.4 \pm 0.1$ \\
\hline $2-0 \mathrm{~S}(7)$ & 1.064 & $0.62 \pm 0.04$ & $1-0 \mathrm{~S}(9)$ & 1.688 & $0.7 \pm 0.1$ \\
\hline $2-0 \mathrm{~S}(6)$ & 1.073 & $0.4 \pm 0.1$ & $1-0 \mathrm{~S}(8)^{d}$ & 1.715 & $0.6 \pm 0.1$ \\
\hline $2-0 \mathrm{~S}(5)$ & 1.085 & $0.69 \pm 0.05$ & $1-0 \mathrm{~S}(7)^{e}$ & 1.748 & $3.9 \pm 0.1$ \\
\hline $2-0 \mathrm{~S}(4)$ & 1.100 & $0.3 \pm 0.2^{b}$ & $1-0 \mathrm{~S}(6)$ & 1.788 & $1.86 \pm 0.05$ \\
\hline $3-1 S(9)+3-1 S(10)+3-1 S(11)$ & $1.120-1.120-1.121$ & $1.4 \pm 0.5^{b}$ & $1-0 \mathrm{~S}(3)$ & 1.958 & $15.4 \pm 0.2$ \\
\hline $3-1 \mathrm{~S}(7)$ & 1.130 & $0.8 \pm 0.3^{b}$ & $2-1 \mathrm{~S}(4)$ & 2.004 & $0.42 \pm 0.09$ \\
\hline $2-0 \mathrm{~S}(2)$ & 1.138 & $0.6 \pm 0.1$ & $1-0 \mathrm{~S}(2)$ & 2.034 & $4.20 \pm 0.06$ \\
\hline $3-1 S(5)$ & 1.152 & $0.9 \pm 0.1$ & $2-1 S(3)$ & 2.073 & $1.63 \pm 0.04$ \\
\hline $2-0 \mathrm{~S}(1)$ & 1.162 & $1.2 \pm 0.1$ & $1-0 \mathrm{~S}(1)$ & 2.122 & $9.59 \pm 0.05$ \\
\hline $3-1 \mathrm{~S}(3)$ & 1.186 & $0.8 \pm 0.1$ & $2-1 \mathrm{~S}(2)$ & 2.154 & $0.51 \pm 0.05$ \\
\hline $2-0 S(0)+4-2 S(10)$ & $1.189-1.190$ & $0.4 \pm 0.1$ & $3-2 S(3)$ & 2.201 & $0.3 \pm 0.1$ \\
\hline 4-2 S(9) & 1.196 & $0.4 \pm 0.1$ & $1-0 \mathrm{~S}(0)$ & 2.223 & $2.58 \pm 0.05$ \\
\hline $4-2 S(8)+4-2 S(11)$ & $1.198-1.199$ & $0.5 \pm 0.2^{b}$ & $2-1 \mathrm{~S}(1)$ & 2.248 & $1.0 \pm 0.2$ \\
\hline 4-2 S(7) & 1.205 & $0.3 \pm 0.1$ & $2-1 \mathrm{~S}(0)$ & 2.355 & $0.32 \pm 0.08$ \\
\hline $3-1 \mathrm{~S}(2)$ & 1.207 & $0.4 \pm 0.1$ & $3-2 S(1)$ & 2.386 & $0.5 \pm 0.1$ \\
\hline $4-2 S(5)$ & 1.226 & $0.4 \pm 0.1$ & $1-0 \mathrm{Q}(1)$ & 2.407 & $10.9 \pm 0.3$ \\
\hline $3-1 \mathrm{~S}(1)$ & 1.233 & $0.5 \pm 0.1$ & $1-0 \mathrm{Q}(2)$ & 2.413 & $4.5 \pm 0.4$ \\
\hline $2-0 \mathrm{Q}(1)$ & 1.238 & $0.55 \pm 0.05$ & $1-0 \mathrm{Q}(3)$ & 2.424 & $9.6 \pm 0.4$ \\
\hline $2-0 \mathrm{Q}(2)+4-2 \mathrm{~S}(4)$ & $1.242-1.242$ & $0.39 \pm 0.04$ & $1-0 \mathrm{Q}(4)$ & 2.437 & $4.2 \pm 0.5$ \\
\hline $2-0 \mathrm{Q}(3)^{c}$ & 1.247 & $0.56 \pm 0.04$ & $1-0 \mathrm{Q}(5)$ & 2.455 & $3.2 \pm 0.5$ \\
\hline $2-0 \mathrm{O}(3)$ & 1.335 & $0.39 \pm 0.07$ & $1-0 \mathrm{Q}(6)$ & 2.476 & $2.2 \pm 0.4$ \\
\hline $3-1 \mathrm{Q}(5)+4-2 \mathrm{~S}(0)$ & $1.342-1.342$ & $0.5 \pm 0.2^{b}$ & $1-0 \mathrm{Q}(7)$ & 2.500 & $6.2 \pm 0.6$ \\
\hline \multicolumn{6}{|c|}{ Ionic lines } \\
\hline$[\mathrm{CI}]{ }^{1} \mathrm{D}_{2}-{ }^{3} \mathrm{P}_{1}$ & 0.983 & $0.98 \pm 0.06$ & {$[\mathrm{Fe}$ II $] \mathrm{a}^{4} \mathrm{D}_{5 / 2}-\mathrm{a}^{6} \mathrm{D}_{5 / 2}$} & 1.295 & $1.4 \pm 0.2$ \\
\hline$[\mathrm{CI}]{ }^{1} \mathrm{D}_{2}-{ }^{3} \mathrm{P}_{2}$ & 0.985 & $2.33 \pm 0.05$ & {$[\mathrm{Fe}$ II $] \mathrm{a}^{4} \mathrm{D}_{7 / 2}-\mathrm{a}^{6} \mathrm{D}_{7 / 2}$} & 1.321 & $2.9 \pm 0.2$ \\
\hline$[\mathrm{S} \mathrm{II}]{ }^{2} \mathrm{P}_{3 / 2}-{ }^{2} \mathrm{D}_{3 / 2}$ & 1.029 & $0.21 \pm 0.07$ & {$[\mathrm{Fe}$ II $] \mathrm{a}^{4} \mathrm{D}_{5 / 2}-\mathrm{a}^{6} \mathrm{D}_{3 / 2}$} & 1.328 & $0.6 \pm 0.1$ \\
\hline$[\mathrm{S} \mathrm{II}]{ }^{2} \mathrm{P}_{3 / 2}-{ }^{2} \mathrm{D}_{5 / 2}$ & 1.032 & $0.30 \pm 0.07$ & {$[\mathrm{Fe}$ II $] \mathrm{a}^{4} \mathrm{D}_{5 / 2}-\mathrm{a}^{4} \mathrm{~F}_{9 / 2}$} & 1.534 & $1.2 \pm 0.2$ \\
\hline$[\mathrm{S} \mathrm{II}]{ }^{2} \mathrm{P}_{1 / 2}-{ }^{2} \mathrm{D}_{3 / 2}$ & 1.034 & $0.28 \pm 0.09$ & {$[\mathrm{Fe}$ II $] \mathrm{a}^{4} \mathrm{D}_{3 / 2}-\mathrm{a}^{4} \mathrm{~F}_{7 / 2}$} & 1.600 & $0.7 \pm 0.2$ \\
\hline$[\mathrm{S} \mathrm{II}]{ }^{2} \mathrm{P}_{1 / 2}-{ }^{2} \mathrm{D}_{5 / 2}$ & 1.037 & $0.2 \pm 0.1^{b}$ & {$[\mathrm{Fe}$ II $] \mathrm{a}^{4} \mathrm{D}_{7 / 2}-\mathrm{a}^{4} \mathrm{~F}_{9 / 2}$} & 1.644 & $8.2 \pm 0.1$ \\
\hline $\mathrm{HeI} 2^{3} \mathrm{~S}-2^{3} \mathrm{P}^{0}$ & 1.083 & $0.53 \pm 0.05$ & {$[\mathrm{Fe}$ II $] \mathrm{a}^{4} \mathrm{D}_{1 / 2}-\mathrm{a}^{4} \mathrm{~F}_{5 / 2}$} & 1.664 & $0.28 \pm 0.07$ \\
\hline $\mathrm{Pa} \gamma$ & 1.094 & $<0.8^{f}$ & {$[\mathrm{Fe}$ II $] \mathrm{a}^{4} \mathrm{D}_{5 / 2}-\mathrm{a}^{4} \mathrm{~F}_{7 / 2}$} & 1.678 & $0.58 \pm 0.08$ \\
\hline$[\mathrm{Fe}$ II $] \mathrm{a}^{4} \mathrm{D}_{7 / 2}-\mathrm{a}^{6} \mathrm{D}_{9 / 2}$ & 1.257 & $8.7 \pm 0.1$ & {$[\mathrm{Fe}$ II $] \mathrm{a}^{4} \mathrm{D}_{3 / 2}-\mathrm{a}^{4} \mathrm{~F}_{3 / 2}$} & 1.798 & $0.4 \pm 0.1$ \\
\hline$[\mathrm{Fe}$ II $] \mathrm{a}^{4} \mathrm{D}_{3 / 2}-\mathrm{a}^{6} \mathrm{D}_{3 / 2}$ & 1.279 & $1.1 \pm 0.2$ & {$[\mathrm{Fe}$ II $] \mathrm{a}^{4} \mathrm{D}_{7 / 2}-\mathrm{a}^{4} \mathrm{~F}_{7 / 2}$} & 1.810 & $3.4 \pm 0.4$ \\
\hline $\mathrm{Pa} \beta$ & 1.283 & $1.5 \pm 0.4$ & $\mathrm{Br} \gamma$ & 2.166 & $<0.2^{f}$ \\
\hline
\end{tabular}

Notes: ${ }^{a}$ The uncertainty refers to the rms of the local baseline. ${ }^{b}$ Signal-to-noise ratio between 2 and $3 .{ }^{c}$ Blended with $[\mathrm{Fe} I \mathrm{II}] \mathrm{a}^{4} \mathrm{D}_{3 / 2}-\mathrm{a}^{6} \mathrm{D}_{5 / 2}(\lambda=$ $1.248 \mu \mathrm{m}) .{ }^{d}$ Blended with [Fe II $] \mathrm{a}^{4} \mathrm{D}_{3 / 2}-\mathrm{a}^{4} \mathrm{~F}_{5 / 2}(\lambda=1.711 \mu \mathrm{m}) .{ }^{e}$ Blended with [Fe II $] \mathrm{a}^{4} \mathrm{P}_{3 / 2}-\mathrm{a}^{4} \mathrm{D}_{7 / 2}(\lambda=1.749 \mu \mathrm{m}) .{ }^{f} 3$ sigma upper limit.

at $2.12 \mu \mathrm{m}$ and the area over which the photometry has been computed. Since fluxes of mid-infrared lines are poorly affected by the extinction (which is in any case low, as will be shown in Sect. 3.1), their ratios directly give a precise indication of the local ortho-to-para ratio. Based on ISO/SWS observations, Neufeld et al. (1998) estimate a value of 1.2 averaged over all the region, while Neufeld et al. (2006) derive values from 0.4 to 2 in knots $\mathrm{C}$ and $\mathrm{E}, \mathrm{K}$, which they interpret as a legacy of an earlier stage when the gas had reached equilibrium at a temperature $\$ 90 \mathrm{~K}$. Disregarding the ratio involving the $5.5 \mu \mathrm{m}$ line, whose image seems to be affected by calibrations problems (as also suggested by the Boltzmann diagram, see Fig. 5), we measure ortho-to-para ratios of $0.9,1.3$ and 2.3 , along the jet direction, from knots $\mathrm{C}$ to $\mathrm{A}$. These values, which are in good agreement with both the estimates of Neufeld et al. (1998) and Neufeld et al. (2006) could be interpreted in terms of subsequent shock heating events due to an episodic jet.

\subsubsection{Spectroscopy}

The 43-197 $\mu$ m spectrum of HH54 was obtained with the Long Wavelength Spectrometer (LWS) aboard ISO and reported by
Table 3. Medium-resolution spectroscopy parameters.

\begin{tabular}{cccc}
\hline \hline Knot & slit & $\begin{array}{c}v-v_{\text {cloud }^{a}}{ }^{a} \\
\left(\mathrm{~km} \mathrm{~s}^{-1}\right)\end{array}$ \\
\hline C1 & III & -15 & $19 \pm 5$ \\
B & III & -12 & $13 \pm 5$ \\
A & III & -12 & $16 \pm 5$ \\
C2 & IV & -14 & $12 \pm 8$ \\
L & IV & -13 & $20 \pm 6$ \\
E & IV & -12 & $13 \pm 7$ \\
K & IV & -9 & $21 \pm 8$ \\
\hline
\end{tabular}

Note: ${ }^{a}$ The estimated uncertainty is within $3 \mathrm{~km} \mathrm{~s}^{-1}$.

Nisini et al. (1996) and Liseau et al. (1996). The spectrum was taken at the beginning of the ISO mission (orbit \#92) and originally processed with a preliminary version of the Offline Processing Software (OLP). Recently, ISO telemetry was reprocessed (with the OLP10.1) and the resulting Relative Spectral Response Function (RSRF) was higher than in the earlier versions; here we report the analysis of this upgraded version of the spectrum. The observation was carried out with the LWS AOT01 $(\lambda / \Delta \lambda \sim 200)$, adopting the "fast scanning" option and an 

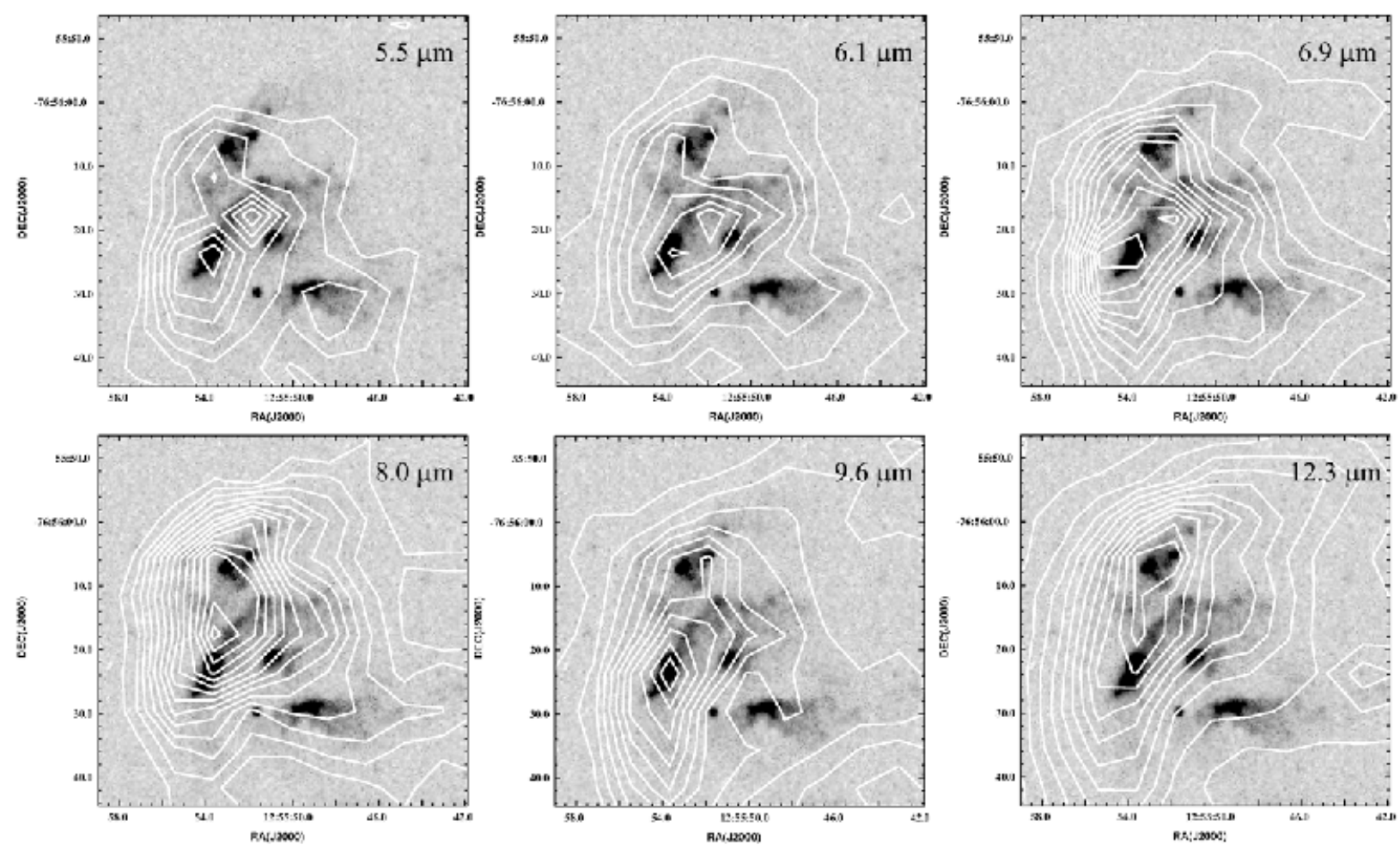

Fig. 3. ISOCAM images of HH54. Contours are in steps of $2 \sigma$ from a $3 \sigma$ level of $8.3 \times 10^{-16} \mathrm{erg} \mathrm{s}^{-1} \mathrm{~cm}^{-2} \operatorname{arcsec}^{-1}$ (at $5.5 \mu \mathrm{m}, 6.1 \mu \mathrm{m}$ and $6.9 \mu \mathrm{m}$ ), $2.7 \times 10^{-16} \mathrm{erg} \mathrm{s}^{-1} \mathrm{~cm}^{-2} \operatorname{arcsec}^{-1}$ (at $\left.8.0 \mu \mathrm{m}\right), 1.4 \times 10^{-15} \mathrm{erg} \mathrm{s}^{-1} \mathrm{~cm}^{-2} \operatorname{arcsec}^{-1}$ (at $9.6 \mu \mathrm{m}$ ) and $5.5 \times 10^{-16} \mathrm{erg} \mathrm{s}^{-1} \mathrm{~cm}^{-2} \operatorname{arcsec}^{-1}($ at $12.3 \mu \mathrm{m})$.

Table 4. Photometry of (0-0) lines observed with ISOCAM.

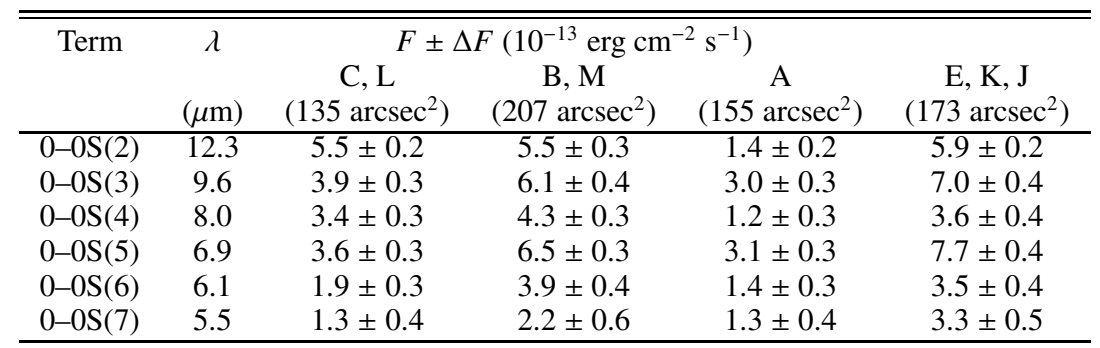

Note: Due to transient effects at low signal levels, the fluxes could be underestimated by a factor 1.3-1.6 (relative fluxes, excitation temperatures, and $\mathrm{o} / \mathrm{p}$ ratios would remain unaffected).

oversampling of 4 times the spectral resolution element. The flux calibration, based on Uranus observations, has an uncertainty of up $30 \%$, while the accuracy on the wavelength calibration is about $25 \%$ of the spectral element $(0.29 \mu \mathrm{m}$ and $0.60 \mu \mathrm{m}$ for the short [43-90 $\mu \mathrm{m}$ ] and long [90-197 $\mu \mathrm{m}$ ] wavelength range, respectively). Post-pipeline processing, which consists of glitch removal, averaging of different spectral scans, and correction for interference fringes, was performed with the ISO Spectral Analysis Package $^{3}$ (ISAP), v.2.1. In comparison with the values reported by Nisini et al. (1996), the new line fluxes, given in Table 5, are systematically 30-40\% lower. In addition, two more lines (CO 20-19 at $130.4 \mu \mathrm{m}$ and $\mathrm{OH}$ at $163 \mu \mathrm{m}$ ) have been detected, but we do not observe the CO 19-18 line at $137 \mu \mathrm{m}$, which was just detectable in the old version of the spectrum (shown by Liseau et al. 1996). The low resolution data have been complemented by the intermediate resolution spectrum of

3 The ISO Spectral Analysis Package ISAP is a joint development by the LWS and SWS Instrument Teams and Data Centers. Contributing institutes are CESR, IAS, IPAC, MPE, RAL and SROAN. the [O I] $63 \mu \mathrm{m}$ line (see Fig. 4). This was accomplished by coupling the LWS with a Fabry-Perot interferometer, thus achieving a resolution of $\approx 8500$ (Swinyard et al. 1996). The line, detected with a signal-to-noise ratio of about five, peaks at $v-v_{\text {cloud }}=$ $-12 \mathrm{~km} \mathrm{~s}^{-1}$, similar to that of the $2.12 \mu \mathrm{m}$ line. Although this could suggest a similar origin for oxygen and molecular hydrogen emission, the $63 \mu \mathrm{m}$ profile is $\approx 39 \mathrm{~km} \mathrm{~s}^{-1}$ wide, and the corresponding FWZI, computed on the line fit by applying the procedure of Hartigan et al. (1987), is $\approx 80 \mathrm{~km} \mathrm{~s}^{-1}$. This value can only be considered a rough estimate, given the poor signalto-noise ratio of the detection, nevertheless it indicates that the $63 \mu \mathrm{m}$ emission may arise in a shock with velocity larger than (or close to) the $\mathrm{H}_{2}$ dissociation limit.

\section{Analysis and discussion}

\subsection{Boltzmann diagrams}

Analysis of the $\mathrm{H}_{2}$ line emission gives rise to estimates for the reddening towards the encompassed knots and the temperature 
Table 5. Far infrared lines observed in HH54.

\begin{tabular}{cccc}
\hline \hline Term & $\begin{array}{c}\lambda_{\text {obs }} \\
(\mu \mathrm{m})\end{array}$ & $\begin{array}{c}\lambda_{\text {vac }} \\
(\mu \mathrm{m})\end{array}$ & $\begin{array}{c}F \pm \Delta F \\
\left(10^{-13} \mathrm{erg} \mathrm{s}^{-1} \mathrm{~cm}^{-2}\right)\end{array}$ \\
\hline$[\mathrm{O} \mathrm{I}]^{3} P_{1}-{ }^{3} P_{2}$ & 63.18 & 63.18 & $102 \pm 2$ \\
$\mathrm{o}-\mathrm{H}_{2} \mathrm{O} 2_{21}-1_{10}$ & $108.07^{b}$ & 108.07 & $<1.2$ \\
$\mathrm{CO} 23-22+\mathrm{o}_{2} \mathrm{H}_{2} \mathrm{O} 4_{14}-3_{03}$ & 113.54 & $113.458-113.537$ & $1.3 \pm 0.4$ \\
$\mathrm{OH}^{2} \Pi_{3 / 2,5 / 2}-{ }^{2} \Pi_{3 / 2,3 / 2}$ & 119.45 & $119.23-119.44$ & $3.0 \pm 0.3$ \\
$\mathrm{CO} 20-19^{a}$ & 130.40 & 130.37 & $0.9 \pm 0.3$ \\
$\mathrm{CO} 19-18$ & $137.20^{b}$ & 137.20 & $<1$ \\
$\mathrm{p}-\mathrm{H}_{2} \mathrm{O} 3_{13}-2_{02}$ & 138.57 & 138.53 & $1.0 \pm 0.3$ \\
$\mathrm{CO} 18-17$ & $144.78^{c}$ & 144.78 & $2.1 \pm 0.3$ \\
{$[\mathrm{O} \mathrm{I}]^{3} P_{0}-{ }^{3} P_{1}$} & $145.52^{c}$ & 145.52 & $4.5 \pm 0.3$ \\
$\mathrm{CO} 17-16$ & 153.19 & 153.27 & $2.2 \pm 0.3$ \\
{$[\mathrm{C} \mathrm{II}]^{2} P_{3 / 2}{ }^{2} P_{1 / 2}$} & 157.72 & 157.74 & $9.0 \pm 0.4$ \\
$\mathrm{CO} 16-15$ & $162.73^{c}$ & 162.81 & $3.9 \pm 0.2$ \\
$\mathrm{OH}^{2} \Pi_{1 / 2,3 / 2}-{ }^{2} \Pi_{1 / 2,1 / 2}{ }^{a}$ & $163.04^{c}$ & $163.12-163.40$ & $1.5 \pm 0.2$ \\
$\mathrm{CO} 15-14$ & $173.63^{b}$ & 173.63 & $6 \pm 1$ \\
$\mathrm{o}-\mathrm{H}_{2} \mathrm{O} 3_{03}-2_{12}$ & $174.63^{b}$ & 174.63 & $2 \pm 1^{d}$ \\
$\mathrm{o}-\mathrm{H}_{2} \mathrm{O} 2_{12}-1_{01}$ & 179.53 & 179.53 & $7 \pm 1$ \\
$\mathrm{CO} 14-13$ & 185.85 & 186.00 & $6 \pm 1$ \\
\hline
\end{tabular}

Notes: ${ }^{a}$ Not detected by Nisini et al. (1996); ${ }^{b}$ fixed parameter; ${ }^{c}$ deblended lines; ${ }^{d}$ signal to noise ratio less than 3.

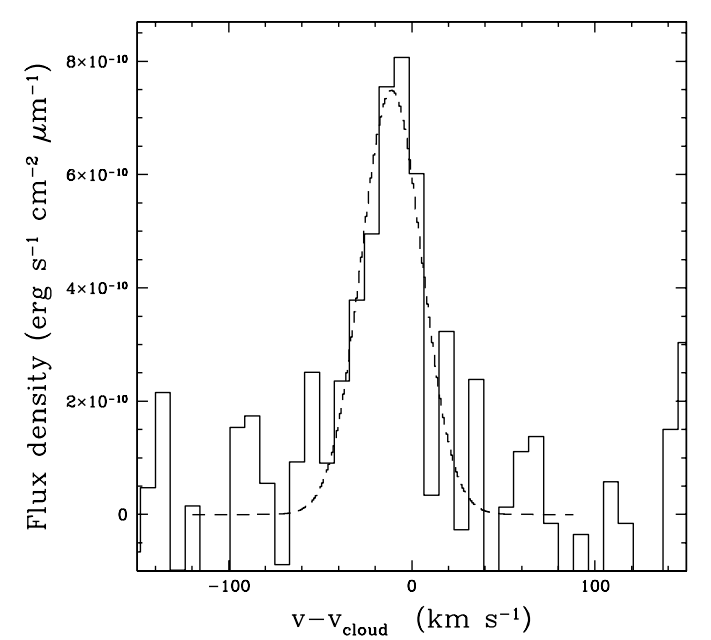

Fig. 4. LWS-FP spectrum of the $[\mathrm{O} \mathrm{I}] 63 \mu \mathrm{m}$ line. The Gaussian fit is shown as a dotted line.

of the emitting gas. These two quantities can be simultaneously estimated in the framework of the Boltzmann (or excitation) diagram, which is a plot of $\ln \left(N_{v J} / g_{J}\right)$ against $E_{v J} / k$, where $N_{v J}\left(\mathrm{~cm}^{-2}\right)$ is the column density of level $(v, J), E_{v J} / k(\mathrm{~K})$ its excitation energy and $g_{J}=(2 J+1)(2 I+1)$ its statistical weight. The nuclear spin quantum number is $I=1$ for ortho- $\mathrm{H}_{2}$ and $I=0$ for para- $\mathrm{H}_{2}$. If the gas is thermalized at a single temperature, the points in the diagram fall onto a straight line: a best fit (by minimization of the deviation of the data-points from the line) to the gradient provides the reciprocal of the gas temperature. At the same time, under the assumption of optically thin emission, the extinction can be evaluated by noting that transitions from the same upper level should lie at the same point in the diagram (e.g. G94). Thus, having adopted the reddening law by Rieke \& Lebofsky (1985), we have tuned the $A_{\mathrm{V}}$ value to maximize the overlap of data points corresponding to lines originating in the same upper level. We derive $A_{\mathrm{V}}=1 \mathrm{mag}$ over all of the region.

In a shock wave there is a range of kinetic temperatures and the Boltzmann diagram forms a curve. In order, therefore, to probe our observations at different temperatures, columns of the pure rotational lines are plotted in a Boltzmann diagram together with those of near-infrared ro-vibrational lines. Since the region emitting in $0-0$ lines has been separated in four distinct zones (as described in Sect. 2.2.1), we have constructed four Boltzmann diagrams, plotted in Fig. 5; in each zone, the column densities of the ro-vibrational lines have been computed by assuming an emitting region defined from the $3 \sigma$ contours of the $2.12 \mu \mathrm{m}$ line. The similarity of the four Boltzmann diagrams in Fig. 5 implies that the excitation conditions are broadly the same for all of the knots in the HH54 region, at least at the spatial resolution $(\approx$ ten $\operatorname{arcsec})$ characteristic of the four zones. Each diagram indicates the presence of two temperature components. A "cold" component at $T \approx 800 \mathrm{~K}$ is traced by the 0-0 lines: this is in substantial agreement with that measured by Neufeld et al. (1998) and Cabrit et al. (1999) on the basis of SWS and CAM observations $(\approx 650 \mathrm{~K})$. All the other lines appear thermalized at about $\sim 3000 \mathrm{~K}$ from north to south (knots $\mathrm{C}$ to A) and at $\sim 2500 \mathrm{~K}$ on the wing; noticeably, this latter result could simply reflect the lack along the wing direction of spectroscopic data in the $J$ band, where $\mathrm{H}_{2}$ lines with high excitation energy (i.e. those tracing hotter temperature components), are located (Giannini et al. 2002). In the plot, the ortho-to-para ratio was assumed equal to the statistical equilibrium value of three: any significant departure from this value should result in a systematic displacement of the ortho with respect to the para levels. While such behaviour is observed among the $v=0$ lines (see also Table 4), no significant deviations from the equilibrium value are appreciable in the data points tracing the hotter gas components (those with $v \geq 1$ ). This can more easily be seen by taking ratios of fluxes of bright ortho and para lines belonging to the same manifold (e.g. those among 1-0 and 2-1 lines, see Table 2), for which the extinction correction is $20 \%$ at worst.

\subsection{Models of molecular emission}

In the previous section we showed that the observed emission is quantitatively similar in both the jet and the wing of HH54, indicating common excitation mechanisms and similar physical conditions for the whole region. Accordingly, we can examine together the $\mathrm{H}_{2}$ emission with the emission of the other molecular species $\left(\mathrm{CO}, \mathrm{H}_{2} \mathrm{O}\right.$ and $\left.\mathrm{OH}\right)$, which are observed with a beam encompassing the whole HH54 region. Furthermore, 


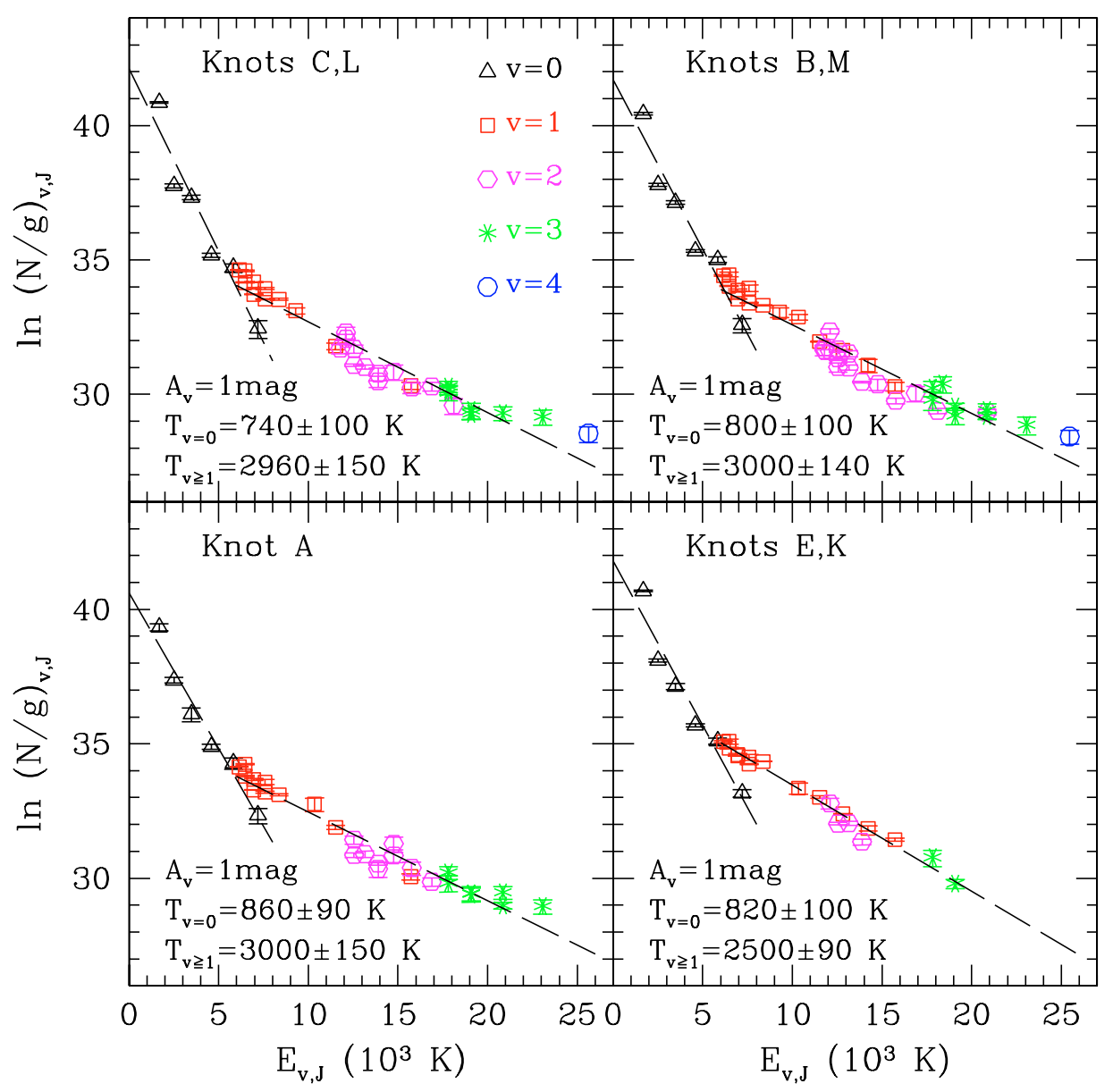

Fig. 5. Boltzmann diagram of the four zones identified in the (0-0) images (see text). In the upper-left panel, symbols adopted to mark different vibrational levels are shown. In each diagram, the derived extinction, along with the temperatures of the vibrational manifolds, are indicated.

we have taken the zone encompassing knots $\mathrm{B}$ and $\mathrm{M}$ (hereafter the "B-zone"), in which $\mathrm{H}_{2}$ is emitted over the widest range of energies observed, to be representative of the excitation conditions in HH54. To explore the diagnostic capabilities of the observed lines in disentangling different shock structures and physical parameters, we have analyzed the observations in the context of a full range of shock models, including steady-state non-dissociative (C-type) and dissociative (J-type) shocks and quasi-steady J-type shocks with magnetic precursor (denoted C, $\mathrm{J}$ and $\mathrm{C}+\mathrm{J}$, respectively, for brevity). For each type of shock we have proceeded in the following way: $i$ ) the $\mathrm{H}_{2}$ column densities in the B-zone were fitted using a shock model, which is described in Sect. 3.2.1; ii) temperature and density profiles along with local velocity gradient predicted by each best-fitting model were used as input parameters for a Large Velocity Gradient (LVG) code to reproduce the $\mathrm{H}_{2} \mathrm{O}, \mathrm{CO}$ and $\mathrm{OH}$ FIR line emission (see Sect. 3.2.3). At this step we can both test the overall consistency of the selected models and also identify the FIR lines most useful in diagnosing the shock physics; iii) finally, in order to determine if atomic/ionic and molecular emission are excited under the same conditions, the O I, C I, S II and Fe II lines fluxes predicted by the best shock model found for the molecular lines were compared with the NIR observations (Sect. 3.2.4).

\subsection{1. $\mathrm{H}_{2}$ emission}

The shock code, MHD_voDE, has been described by Le Bourlot et al. (2002) and Flower et al. (2003), while the method used to calculate the $\mathrm{H}_{2}$ emission has been explained by Giannini et al. (2004) and $\mathrm{M}^{\mathrm{c}}$ Coey et al. (2004). Here, only the aspects most relevant to this study are reviewed.

The code solves for one-dimensional, planar, multi-fluid flow and can simulate both steady-state J- and C- type shock waves and quasi-steady J-type shocks with magnetic precursor. The differential equations that determine the abundances of the chemical species are solved in parallel with the magnetohydrodynamical conservation equations for the neutral, positively and negatively charged fluids.

The calculations for the distribution of population among $150 \mathrm{H}_{2}$ ro-vibrational levels (i.e. up to an energy of $3.9 \times 10^{4} \mathrm{~K}$ ) take into account collisional excitation and de-excitation, spontaneous radiative decay, collisional dissociation and ionization, and reformation of $\mathrm{H}_{2}$ on grains, and are solved in parallel with the chemical and dynamical conservation equations. This approach is essential to ensure the accuracy of the computed $\mathrm{H}_{2}$ column densities, because the level populations do not respond instantaneously to changes in the physical state of the gas.

The column density of a level is sensitive to the pre-shock density, the shock velocity, the age of the shock and the strength of the magnetic field. The response of the level populations to these variables, which are parameters of the code, can be seen in the excitation diagram. If the gas is in local thermodynamic equilibrium (LTE), the Boltzmann plot is a smooth curve, with little scatter of the points about the median line. Departures from LTE enhance the scatter. Increases in the shock velocity, $v_{\mathrm{s}}$, and the pre-shock density, $n_{\mathrm{H}}$, act to increase the rate of 
collisions that vibrationally excite the $\mathrm{H}_{2}$ molecules and thus decrease the departure from LTE. The presence of a magnetic field acts to dampen and broaden the shock wave; therefore, an increased magnetic field strength enhances the departure from LTE. The magnetic field is assumed to scale by a factor $b$ with the square root of the gas density and is expressed as $B(\mu \mathrm{G})=$ $b\left[n_{\mathrm{H}}\left(\mathrm{cm}^{-3}\right)\right]^{0.5}$. The assumed dependence of $B$ on $n_{\mathrm{H}}$ derives from considerations of energy equipartition: the magnetic energy density is $\propto B^{2}$, whereas the thermal energy density is $\propto n$. We note that the shock front is assumed to be planar, and the parameter $b$ scales the component of the magnetic field parallel to the shock front (and perpendicular to the flow direction), consonant with the assumed dependence of $B$ on $n_{\mathrm{H}}$. Thus, $n_{\mathrm{H}}=10^{4} \mathrm{~cm}^{-3}, b=1$ corresponds to a transverse magnetic field strength $B=100 \mu \mathrm{G}$.

The evolutionary age of the shock wave has a marked effect on the excitation diagram. The younger the object, the closer is the shock wave to being pure J-type and the higher are the column densities of the more highly excited ro-vibrational levels. Furthermore, the populations of these levels tend to be closer to LTE. In a J-type shock with a magnetic precursor, the levels of $v=0,1$ are populated principally in the precursor and exhibit greater departures from LTE than the higher levels. Furthermore, most of the population remains in the $v=0$ ground vibrational state due to radiative cascade from excited vibrational states.

The code was systematically applied over a grid of the above parameters for each category of shock $(\mathrm{C}, \mathrm{J}$ and $\mathrm{C}+\mathrm{J})$ as follows: $10^{3} \mathrm{~cm}^{-3} \leq n_{\mathrm{H}} \leq 10^{5} \mathrm{~cm}^{-3} ; 1 \mathrm{~km} \mathrm{~s}^{-1} \leq v_{\mathrm{s}} \leq v_{\text {crit }}$ (in the case of C-type shocks), where $v_{\text {crit }}$ is the shock velocity at which a steady-state C-type shock becomes discontinuous due to $\mathrm{H}_{2}$ dissociation (Le Bourlot et al. 2002); $0 \mu \mathrm{G} \leq B \leq 500 \mu \mathrm{G}$; and, for ages greater than that at which a J-type shock with a magnetic precursor becomes indistinguishable from a J-type shock wave (approximately $100 \mathrm{yr}$ for $n_{\mathrm{H}}=10^{4} \mathrm{~cm}^{-3}$ and $v_{\mathrm{s}}=$ $\left.18 \mathrm{~km} \mathrm{~s}^{-1}\right)$.

From rotational $\mathrm{H}_{2}$ transitions observed towards HH54, Neufeld et al. (1998) derived a pre-shock ortho-to-para ratio of $\leq 0.2$. We investigated the effect of the initial ortho-to-para ratio on the Boltzmann diagram and found that, indeed, a value of $\leq 0.2$ is required in order to reproduce the populations in the $v=0$ levels. If the maximum neutral temperature is sufficiently high (exceeding $1300 \mathrm{~K}$ for C-type shocks. or $10^{4} \mathrm{~K}$ forJ-type shocks) then the local ortho-to-para ratio attains the statistical equilibrium value of 3 (Wilgenbus et al. 2000). This condition is met in the models considered (see Fig. 7) and so the $v>0$ levels, which are populated mostly in the hot gas behind the shock front, are insensitive to the initial ortho-to-para ratio.

For the steady $\mathrm{C}$ and $\mathrm{J}$ shock models, we have integrated line fluxes out to a flow time of $10^{5} \mathrm{yr}$, where the post-shock gas has reached thermo-chemical equilibrium. For the young $\mathrm{C}+\mathrm{J}$ shock, the total flow time through the shock wave should be less than the time to reach steady state for the $\mathrm{C}$ precursor, about $10^{4} \mathrm{yr}$ (otherwise one would have a pure $\mathrm{C}$-shock, not a $\mathrm{C}+\mathrm{J}$ ). However, the exact flow time through the J-cooling zone following the C-precursor is not precisely constrained, as it depends on the detailed shock history (e.g. Lesaffre et al. 2004a,b), and on any time-variability in the inflow conditions. Here we have chosen, for illustrative purposes, to truncate our flux integration at $t_{i}=$ $3000 \mathrm{yr}$, by this time the contribution to the $\mathrm{H}_{2}$ and ISO FIR lines in negligible. We note that that fully time-dependent (and 2D) calculations are required for more detailed predictions.

The first result is that $\mathrm{C}$-shock models are unable to reproduce the $\mathrm{H}_{2}$ emission: a J-type component is required in order to produce the observed degree of thermalisation among
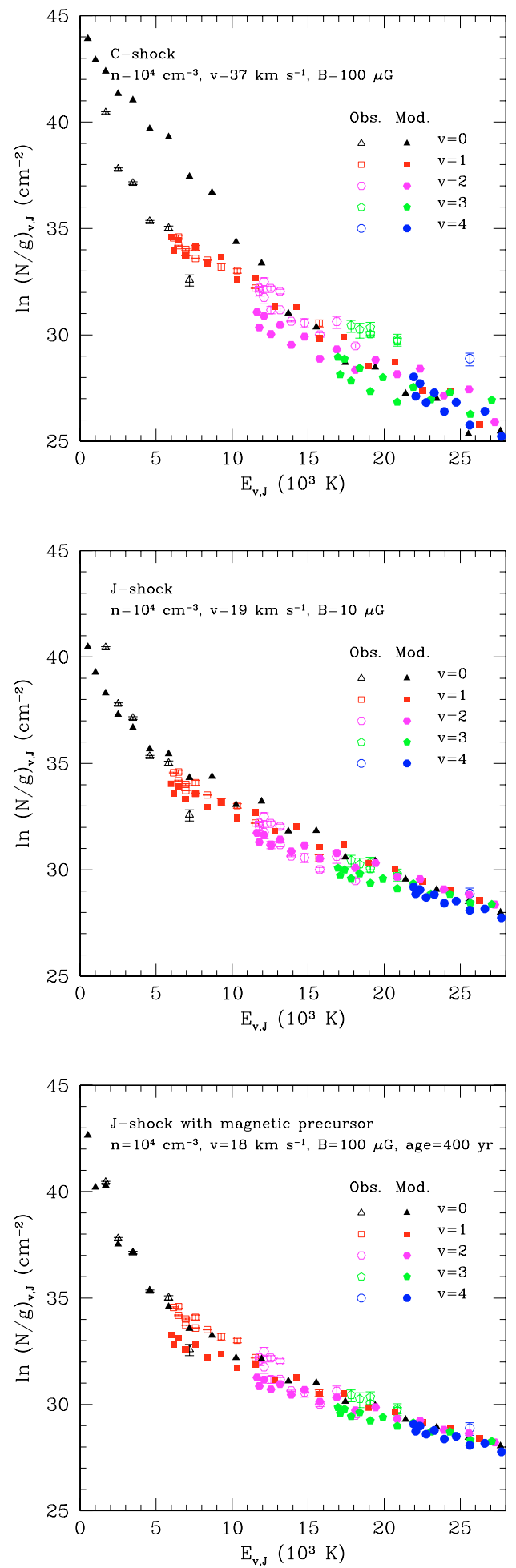

Fig. 6. $\mathrm{H}_{2}$ Boltzmann diagrams of the B-zone compared with the predictions for a steady-state C-type and J-type shocks (top and middle pan$e l s$ ) and for a J-type shock with a magnetic precursor (bottom panel). The shock model parameters are given in each panel and each vibrational state is labelled with a symbol, as indicated.

the levels. As a demonstration, the excitation diagram from the best-fitting steady-state C-type shock model $\left(v_{\mathrm{s}}=37 \mathrm{~km} \mathrm{~s}^{-1}\right.$, $n_{\mathrm{H}}=10^{4} \mathrm{~cm}^{-3}$ ) is plotted along with the observations in Fig. 6 , top panel. This model fits the $v=1$ levels reasonably well but overestimates the lower energy levels while underestimating the higher energy levels. 
Table 6. Parameters of shock models selected, for each category of shocks, as best fits to the $\mathrm{H}_{2}$ emission.

\begin{tabular}{ccccc}
\hline \hline Shock type & $\begin{array}{c}v_{\text {shock }} \\
\left(\mathrm{km} \mathrm{s}^{-1}\right)\end{array}$ & $\begin{array}{c}n_{\mathrm{H}}^{a} \\
\left(\mathrm{~cm}^{-3}\right)\end{array}$ & $\begin{array}{c}\text { Age } \\
(\mathrm{yr})\end{array}$ & $\begin{array}{c}B^{b} \\
(\mu \mathrm{G})\end{array}$ \\
\hline $\mathrm{C}$ & 37 & $10^{4}$ & - & 100 \\
$\mathrm{~J}$ & 19 & $10^{4}$ & - & 10 \\
$\mathrm{C}+\mathrm{J}$ & 18 & $10^{4}$ & 400 & 100 \\
\hline
\end{tabular}

${ }^{a} n_{\mathrm{H}}=n(\mathrm{H})+2 n\left(\mathrm{H}_{2}\right) \cdot{ }^{b} B(\mu \mathrm{G})=b\left[n_{\mathrm{H}}\left(\mathrm{cm}^{-3}\right)\right]^{0.5}, b=1$ for the $\mathrm{C}$ and $\mathrm{C}+\mathrm{J}$ shock models and 0.1 for the $\mathrm{J}$ shock model.

On the other hand, two models were found to produce a good fit to the $\mathrm{H}_{2}$ emission: a J-type shock with a magnetic precursor of age $400 \mathrm{yr}$ and $v_{\text {shock }}=18 \mathrm{~km} \mathrm{~s}^{-1}$ and a steady-state J-type shock with $v_{\text {shock }}=19 \mathrm{~km} \mathrm{~s}^{-1}$. A pre-shock density of $10^{4} \mathrm{~cm}^{-3}$ is predicted by both models. Table 6 and Fig. 7 report the full set of parameters and the velocity and temperature profiles, respectively, while the excitation diagrams produced from these models can be compared with those derived from observations in Fig. 6. The $v>1$ energy levels are fitted well by both models, because the J-component, which is largely responsible for the population of the higher energy levels in the case of the J-type shock with magnetic precursor, is essentially the same in each case (see Fig. 6 middle and bottom panels). The $\mathrm{C}+\mathrm{J}$ shock model reproduces the population in the $v=0$ levels but underestimates the $v=1$ levels. The higher degree of thermalisation arising in the steady-state J-type shock results in a greater population in the $v=1$ levels but this is at the cost of population in the $v=0$ levels and is still insufficient to reproduce the observations, a result which agrees with the findings by Wilgenbus et al. (2000).

Therefore, at this step of the analysis, it can be concluded that a dissociative component is required to fit the $\mathrm{H}_{2}$ emission, even if from the $\mathrm{H}_{2}$ data alone, it is not possible to distinguish between a steady-state J-type shock and a quasi-steady J-type shock with magnetic precursor. It is interesting to note, however, that a model of the latter type could be used to describe the larger scale geometry of the HH54 region. In particular, the smoothness of the bow-shape traced by the rotational lines (Fig. 3), which are excited predominantly in the continuous precursor, in comparison with the clumpiness of the ro-vibrational lines, which arise in the discontinuity and are more affected by the time variability of the driving jet.

\subsubsection{Implications of the derived shock parameters}

We want now briefly to analyze the environmental impact caused by the physical quantities which derive from the shock wave model. Indeed, numerical simulations show that the momentum transfer rate from a high Mach number jet to the ambient medium has a similar efficiency in 1D and 3D (Chernin et al. 1994). Therefore, it is meaningful to compare the momentum transfer rate derived from our 1D shock parameters with that measured in the $\mathrm{CO}$ outflow by K92. With our estimated total shock area of $60^{\prime \prime} \times 60^{\prime \prime}$ (from CO line intensities, see Sect. 3.2.3), a preshock density of $n_{\mathrm{H}}=10^{4} \mathrm{~cm}^{-3}$ and a shock velocity of $18 \mathrm{~km} \mathrm{~s}^{-1}$, we infer a momentum rate of $3 \times$ $10^{-4} M_{\odot} \mathrm{km} \mathrm{s}^{-1} \mathrm{yr}^{-1}$. In comparison, K92 estimated a lower limit of $5 \times 10^{-5} M_{\odot} \mathrm{km} \mathrm{s}^{-1} \mathrm{yr}^{-1}$ in the $\mathrm{CO}$ outflow, which is only a factor of six lower than that derived by our shock model. Consequently, our proposed molecular shock is compatible with the working surface shock driving the whole CO outflow, although we cannot exclude the possibility that other gas
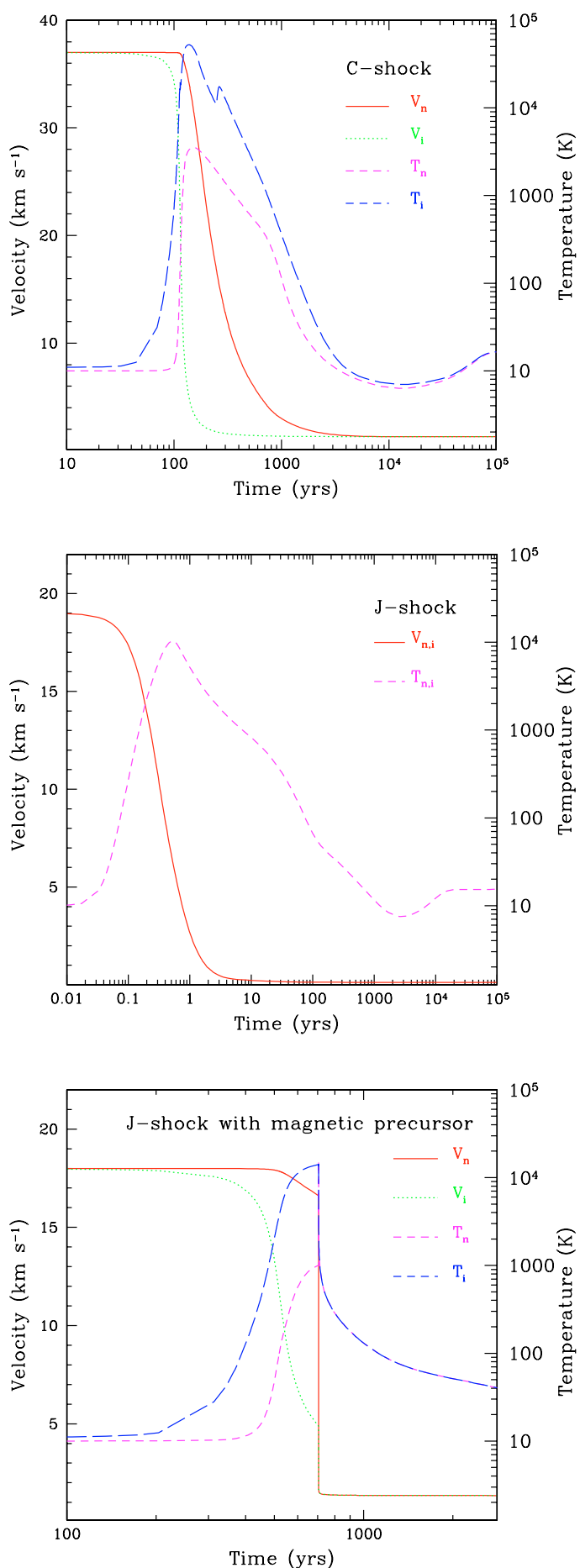

Fig. 7. Velocity and temperature profiles from the models of the bestfitting steady-state C-type and J-type shock (top and middle panels) and the best-fitting J-type shock with a magnetic precursor (bottom panel). The velocity is expressed in the shock frame and the pre-shock gas is to the left.

components (e.g. un-shocked gas, Caratti o Garatti et al. 2006) may exist and thus contribute to the momentum transfer balance.

We can further evaluate the mass-loss rate of the driving engine, if the jet speed and the density ratio between the jet and the ambient medium (which determines the fraction of jet momentum transferred to the cloud; e.g. Chernin et al. 1994) are known. The optical "streamer" south of HH54, which is presumably tracing the "jet" in this system, has a typical radial velocity of 
$-70 \mathrm{~km} \mathrm{~s}^{-1}$ with respect to the cloud (Graham \& Hartigan 1988), hence the jet speed is probably at least $100 \mathrm{~km} \mathrm{~s}^{-1}$. Comparing the atomic line ratios in the streamer measured by the same authors with the 1D models of Hartigan et al. (1994) indicates a jet density $\approx 10^{3} \mathrm{~cm}^{-3}$. Since our $\mathrm{H}_{2}$ shock modelling suggests an ambient molecular cloud density of $10^{4} \mathrm{~cm}^{-3}$, the jet would be strongly under-dense, and the efficiency of momentum transfer to the HH54 shock would be close to 1 . The mass-loss rate in the jet would then be $<3 \times 10^{-4} M_{\odot} \mathrm{km} \mathrm{s}^{-1} \mathrm{yr}^{-1} / 100 \mathrm{~km} \mathrm{~s}^{-1}=$ $3 \times 10^{-6} M_{\odot} \mathrm{yr}^{-1}$.

\subsection{3. $\mathrm{CO}, \mathrm{H}_{2} \mathrm{O}$ and $\mathrm{OH}$ emission}

Aiming to understand the diagnostic capabilities of the farinfrared lines in discriminating different shock types, we have considered all three models described in Table 6, even though steady-state C-type shocks have been already disregarded on the basis of the $\mathrm{H}_{2}$ emission fit. Our 1D assumption imposes a major limitation on the prediction of line profiles, which can be strongly influenced by the geometry; hence we will limit our analysis to the computation of the brightness of the lines. For each model, the physical parameters at each time-step along the shock have been iteratively used as input of a LVG code in planeparallel geometry. This gives as output the brightness of the lines at each temporal step, which have been summed up to obtain the total integrated value to be compared with the observations.

In the LVG approximation, the line ratios are constrained only by the local gas kinetic temperature and density, in addition to the ratio of the species number density and velocity gradient. Hence, in this part of the analysis, we simply compare observed line ratios with those determined by the physical conditions provided by the shock profiles. The unique free parameters are the predicted absolute line brightnesses, whose ratios with the observed fluxes constrain for each molecular species the angular size of the emitting region, and the temperature of the dust, whose continuum emission has the effect of pumping the population of the low lying energy levels. The latter parameter, however, is relevant only for the $\mathrm{OH}$ emission fitting (e.g. Thai-Q-Thung et al. 1998; Offer \& van Dishoeck 1992).

The code has been developed for the first 45 rotational levels for both ortho- and para- $\mathrm{H}_{2} \mathrm{O}, 41$ (and 61) levels for $\mathrm{CO}$ and 24 for $\mathrm{OH}$. Energy levels and radiative decay rates have been taken from the LAMDA database (Leiden Atomic and Molecular Database $)^{4}$. In the $\mathrm{H}_{2} \mathrm{O}$ model, collisions with $\mathrm{p}-\mathrm{H}_{2}$ and $\mathrm{o}-\mathrm{H}_{2}$ are considered separately in the temperature range $5-140 \mathrm{~K}$ (Dubernet \& Grosjean 2002; Grojean et al. 2003; Phillips et al. 1996), while at higher temperatures (up to $2000 \mathrm{~K}$ ) the collisional rates with He have been rescaled to obtain a first order approximation for collisions with $\mathrm{H}_{2}$ (Green et al. 1993). We have assumed an ortho-to-para $\mathrm{H}_{2} \mathrm{O}$ ratio of 3 , as the large uncertainties associated with the observations do not allow us to discriminate among different values of this parameter. In the case of the $\mathrm{OH}$ model we have taken the collisional coefficients with $\mathrm{o}-\mathrm{H}_{2}$ and $\mathrm{p}-\mathrm{H}_{2}$ given by Offer et al. (1994) in the range from 15 to $300 \mathrm{~K}$. Finally, for the CO molecule, we have explored the sensitivity of the model to the different collisional rates available in the literature. McKee et al. (1982) give the $\gamma_{J 0}$ coefficients, scaled from the collisional rates with $\mathrm{He}$, to compute the downward rates for levels with $J_{\text {up }} \leq 60$ and $200 \mathrm{~K} \leq T \leq 2000 \mathrm{~K}$ : this dataset seems therefore appropriate for line predictions in hightemperature regime. The low-temperature regime, on the contrary, has been investigated by Flower (2001) who has computed

\footnotetext{
${ }^{4}$ Available at http://www.strw. leidenuniv.nl/ moldata
}
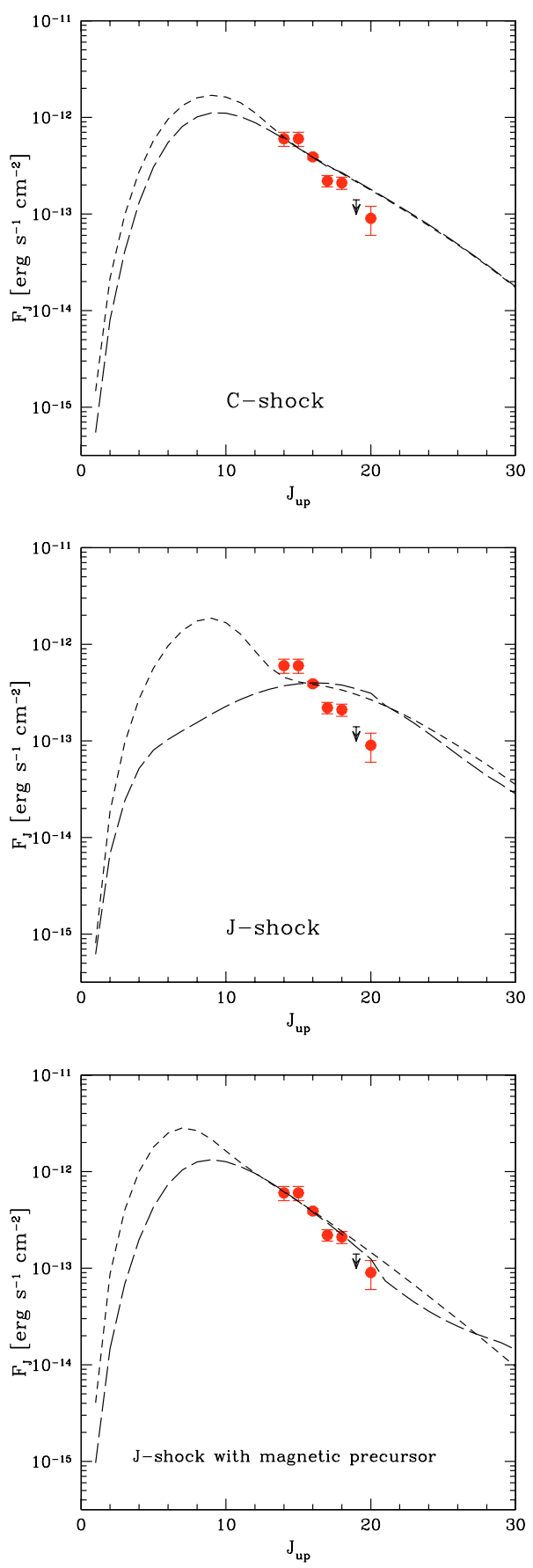

Fig. 8. Top: $\mathrm{CO}$ line fluxes as a function of the rotational quantum number $J_{\text {up }}$. Continuous lines give the fluxes predicted by the C-shock model by assuming the collisional parameters of McKee (1982) (short-dashed) and of Flower (2001) plus Schöier et al. (2005) (long-dashed). The error bars represent the $1 \sigma$ uncertainty. The downward arrow indicates a $3 \sigma$ upper limit; middle: as for the top panel but for the steady-state J-shock; bottom: as for the top panel but for the J-shock with a magnetic precursor.

the collisional rates in the range from 5 to $400 \mathrm{~K}$, including energy levels up to $J_{\text {up }}=29$ and $J_{\text {up }}=20$ for collisions with para$\mathrm{H}_{2}$ and ortho- $\mathrm{H}_{2}$, respectively. Extrapolations to include energy levels up to $J_{\text {up }}=40$ and collisional temperatures up to $2000 \mathrm{~K}$ have been made by Schöier et al. (2005).

The results for the $\mathrm{CO}$ line predictions are plotted in Fig. 8: the long-dashed line refers to the collisional rates from McKee (1982), while the short-dashed one to the rates from 
Flower (2001) and Schöier et al. (2005). Major differences (up to one order of magnitude in the predicted line brightness) occur for $J_{\text {up }} \lesssim 10$, i.e. for levels mainly populated at the low temperatures. This difference is particularly evident in the J-type shock model (middle panel): adopting the coefficients of McKee et al. (1982), we obtain a two-component distribution, with peaks at $J_{\text {up }} \simeq 8$ and $J_{\text {up }} \simeq 16$, while the coefficients of Flower (2001) and Schöier et al. (2005) produce a distribution of fluxes with a single peak at $J_{\text {up }} \simeq 16$. Both sets of coefficients give similar results in the range of the ISO observations, which are rather well fitted by both the steady-state $\mathrm{C}$-type shock and by the $(\mathrm{C}+\mathrm{J})$ shock. On the other hand, the predictions of the steady-state J-type model deviate significantly from the observations. Therefore, we conclude (having already disregarded the $\mathrm{C}$-type shock on the basis of the $\mathrm{H}_{2}$ fit $)$ that the $(\mathrm{C}+\mathrm{J})$ model is the only one able to account for both $\mathrm{H}_{2}$ and $\mathrm{CO}$ observations, a result underlying the importance of a multi-species analysis in modelling line emission from shocks.

The angular size found from the ratio of the theoretical line brightness, from the $(\mathrm{C}+\mathrm{J})$ model, of the $16-15$ line (at $162 \mu \mathrm{m})$ with the observed flux, is about $40^{\prime \prime}$, regardless of the adopted set of coefficients. By considering that the LWS aperture $\left(80^{\prime \prime}\right)$ represents the Airy disk at $\approx 100 \mu \mathrm{m}$ of a point-like source (Swinyard et al. 1996), it follows that the derived dimension is a lower limit to the true size of the emitting region. This latter, taking into account the diffraction effects at $162 \mu \mathrm{m}$, can be estimated to be approximately $60^{\prime \prime}$, i.e. a slightly larger region than that traced by the $\mathrm{H}_{2}$ pure rotational emission. The model indicates that the far-infrared lines arise mainly from gas at $T \approx$ $500-1000 \mathrm{~K}$, which is in agreement with the temperature found from consideration of the $\mathrm{H}_{2} \mathrm{O}-0$ lines in the Boltzmann diagram. Transitions with $6 \lesssim J_{\text {up }} \lesssim 10$ (at wavelengths between 260 and $433 \mu \mathrm{m}$ ) are predicted to be from a few to an order of magnitude brighter than in the other two models: their observation would thus provide a stringent test to the $(\mathrm{C}+\mathrm{J})$ model. This will be possible in the near future with the HIFI spectrometer aboard the Herschel satellite, but also from the ground with the new sub-mm APEX telescope (up to the $J=8-7$ transition). At present, a map of the $\mathrm{CO} 1-0$ emission has been reported by $\mathrm{K} 92$, from which a flux of $\approx 4 \times 10^{-16} \mathrm{erg} \mathrm{s}^{-1} \mathrm{~cm}^{-2}$ is derived by integrating over $\approx 1 \operatorname{arcmin}^{2}$. Noticeably, this value is only a factor of 2.5 lower than that predicted by the $\mathrm{C}+\mathrm{J}$ model, if the coefficients of Flower (2001) and Schöier et al. (2005) are adopted.

Figure 9 reports the fits to the $\mathrm{H}_{2} \mathrm{O}$ ortho and para lines. All three shock models provide good fits to the observed lines, with the marginal exception of the steady-state C-type shock model: the poor diagnostic capability of the LWS water lines is due to their similar excitation temperatures (of $\approx 50-100 \mathrm{~K}$ ). To search for lines more sensitive to the shock parameters, we have reported in the figure the line fluxes predicted for bright $\mathrm{H}_{2} \mathrm{O}$ lines falling in the spectral range covered by the two Herschel spectrometers PACS and HIFI. Indeed, under the physical conditions determined by the considered shock models, some fluxes of lines (mainly at the shortest wavelengths) are predicted to change by more than a factor of two. These, indicated by an asterisk in Table 7, will be all observable with a signal-to-noise ratio larger than ten with an integration time of few minutes with PACS and less than one hour with HIFI (Poglitsch et al. 2001; de Graauw \& Helmich 2001). In the same table, we also report the maximum value of the line optical depth along the $(\mathrm{C}+\mathrm{J})$ shock profile: we note that lines originating in low-lying levels (those with $E_{\text {up }} \lesssim 200 \mathrm{~K}$ ) are optically thick, being the largest $\tau$ values found in correspondence of
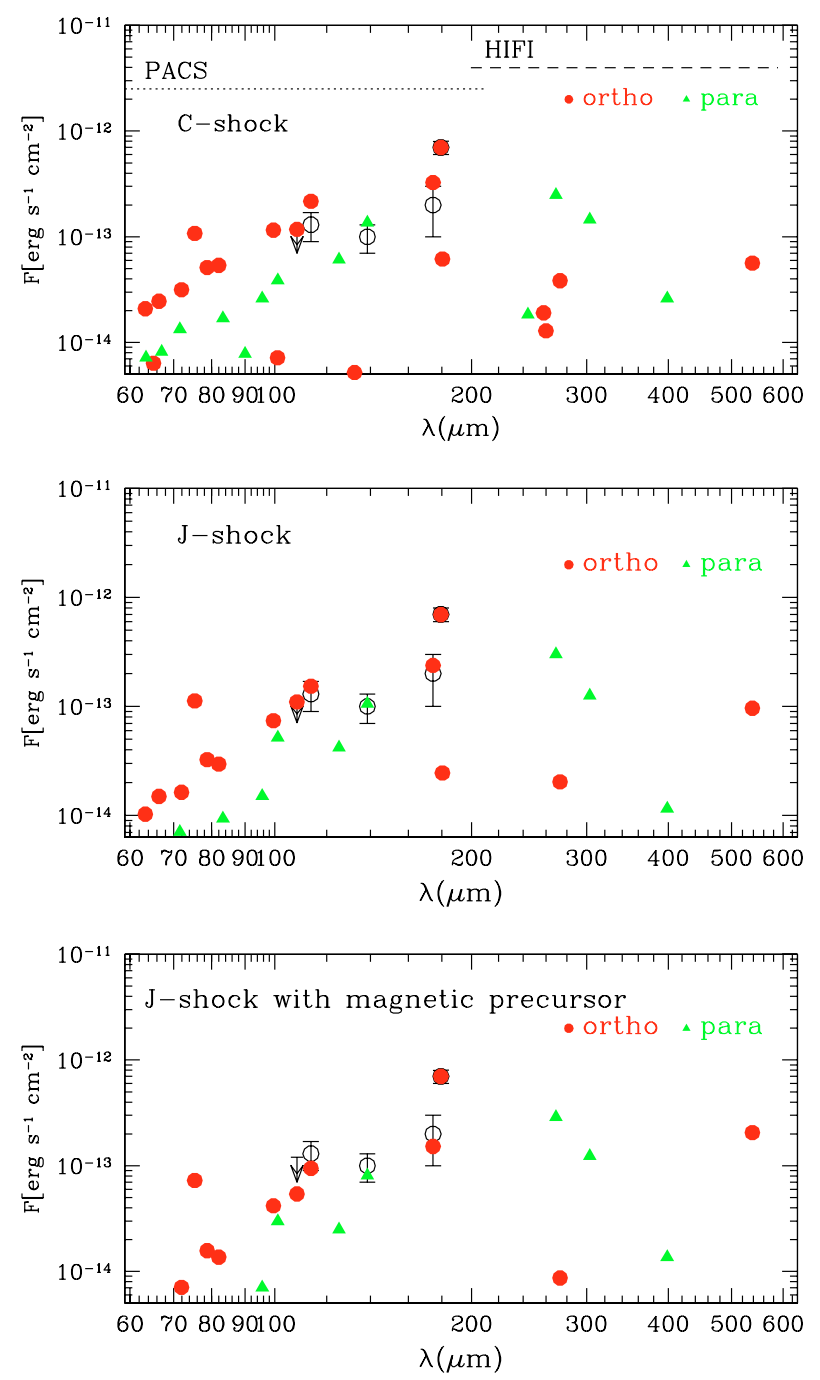

Fig. 9. Top panel: $\mathrm{H}_{2} \mathrm{O}$ line fluxes as a function of wavelength. The LWS spectral range extends up to $200 \mu \mathrm{m}$, but we report in the figures all the lines falling in the range open to Herschel observations. Data points with the relative $1 \sigma$ uncertainties are represented with open circles, while the ortho- and para- $\mathrm{H}_{2} \mathrm{O}$ fluxes predicted by the C-shock model of Table 6 are shown with filled circles and triangles, respectively. The downward arrow indicates a $3 \sigma$ upper limit. The wavelength range covered by PACS and HIFI is indicated, as well. Middle panel: as in the top panel but for the steady-state J-shock model. Bottom panel: as in the top panel but for the J-shock with a magnetic precursor model.

backbone transitions. We also show in Fig. 10 (top panel) the brightness and optical depth of the $179 \mu \mathrm{m}$ line predicted by the $(\mathrm{C}+\mathrm{J})$ model. The line brightness increases to its final value of $\sim 10^{-4} \mathrm{erg} \mathrm{cm}^{-2} \mathrm{~s}^{-1} \mathrm{sr}^{-1}$ with the sudden increase of the temperature at the J-shock front; a corresponding decrease in the optical depth is seen at the same point. In the bottom panel, the water column density and abundance are plotted: the latter increases at the shock front because of the activation of very efficient high-temperature reactions (at $T \gtrsim 300 \mathrm{~K}$ ), which convert all oxygen not locked in $\mathrm{CO}$ into water (e.g. Bergin et al. 1998). In the post-shock region, $N\left(\mathrm{H}_{2} \mathrm{O}\right)$ levels out at $\approx 6 \times 10^{16} \mathrm{~cm}^{-2}$. At the same time, the average abundance $\left\langle x\left(\mathrm{H}_{2} \mathrm{O}\right)\right\rangle$ over the far-IR emitting region, determined as the ratio $N\left(\mathrm{H}_{2} \mathrm{O}\right) / N\left(\mathrm{H}_{2}\right)$ integrated over the shock up to $3 \times$ $10^{3} \mathrm{yr}$, is $\sim 7 \times 10^{-5}$. This roughly agrees with the estimates by Liseau et al. (1996) and also with the values found in a number of outflows from Class 0 sources (Giannini et al. 2001). Thus, 
Table 7. $\mathrm{H}_{2} \mathrm{O}$ fluxes as predicted by the selected shock models.

\begin{tabular}{ccccccc}
\hline \hline $\begin{array}{c}\text { Wavelength } \\
(\mu \mathrm{m})\end{array}$ & Term & $\begin{array}{c}E_{\mathrm{up}} \\
\left(\mathrm{cm}^{-1}\right)\end{array}$ & $\mathrm{C}$ & $\begin{array}{c}\mathrm{J} \\
\left(\mathrm{erg} \mathrm{cm}^{-2} \mathrm{~s}^{-1}\right)\end{array}$ & $\mathrm{C}+\mathrm{J}$ & $\tau^{b}$ \\
\hline 75.4 & o- $3_{21}-2_{12}$ & 212.16 & $1.1 \times 10^{-13}$ & $1.1 \times 10^{-13}$ & $7.2 \times 10^{-14}$ & $\sim 10$ \\
$78.7^{*}$ & o- $4_{23}-3_{12}$ & 300.36 & $5.1 \times 10^{-14}$ & $3.2 \times 10^{-14}$ & $1.6 \times 10^{-14}$ & $\ll 1$ \\
$82.0^{*}$ & o- $6_{16}-5_{05}$ & 447.25 & $5.4 \times 10^{-14}$ & $2.9 \times 10^{-14}$ & $1.3 \times 10^{-14}$ & $\ll 1$ \\
$99.5^{*}$ & o- $5_{05}-4_{14}$ & 325.35 & $1.2 \times 10^{-13}$ & $7.5 \times 10^{-14}$ & $4.2 \times 10^{-14}$ & $\ll 1$ \\
$125.3^{*}$ & p- $4_{04}-3_{13}$ & 222.05 & $6.1 \times 10^{-14}$ & $4.2 \times 10^{-14}$ & $2.4 \times 10^{-14}$ & $\sim 1$ \\
138.5 & p- $3_{13}-2_{02}$ & 142.29 & $1.3 \times 10^{-13}$ & $1.0 \times 10^{-13}$ & $8.1 \times 10^{-14}$ & $\sim 10$ \\
174.6 & o- $3_{03}-2_{12}$ & 176.76 & $3.2 \times 10^{-13}$ & $2.4 \times 10^{-13}$ & $1.5 \times 10^{-13}$ & $\sim 10$ \\
179.5 & o- $2_{12}-1_{01}$ & 79.50 & $6.9 \times 10^{-13}$ & $6.9 \times 10^{-13}$ & $6.9 \times 10^{-13}$ & $\sim 10$ \\
$257.8^{*}$ & o- $3_{21}-3_{12}$ & 212.16 & $1.9 \times 10^{-14}$ & $2.5 \times 10^{-15}$ & $1.5 \times 10^{-15}$ & $\ll 1$ \\
269.3 & p- $1_{01}-0_{00}$ & 37.14 & $2.4 \times 10^{-13}$ & $3.0 \times 10^{-13}$ & $2.9 \times 10^{-13}$ & $\sim 5 \times 10^{2}$ \\
398.6 & p- $2_{11}-2_{02}$ & 95.17 & $2.6 \times 10^{-14}$ & $1.1 \times 10^{-14}$ & $1.4 \times 10^{-14}$ & $\sim 1 \times 10^{2}$ \\
$538.3^{*}$ & o- $1_{10}-1_{01}$ & 42.37 & $5.6 \times 10^{-14}$ & $9.6 \times 10^{-14}$ & $2.0 \times 10^{-13}$ & $\sim 3 \times 10^{2}$ \\
\hline
\end{tabular}

Notes: ${ }^{a}$ lines whose flux changes by more than a factor of two, depending from the shock type, are marked with an asterisk; ${ }^{b}$ maximum value of the optical depth along the profile of the $(\mathrm{C}+\mathrm{J})$ shock.

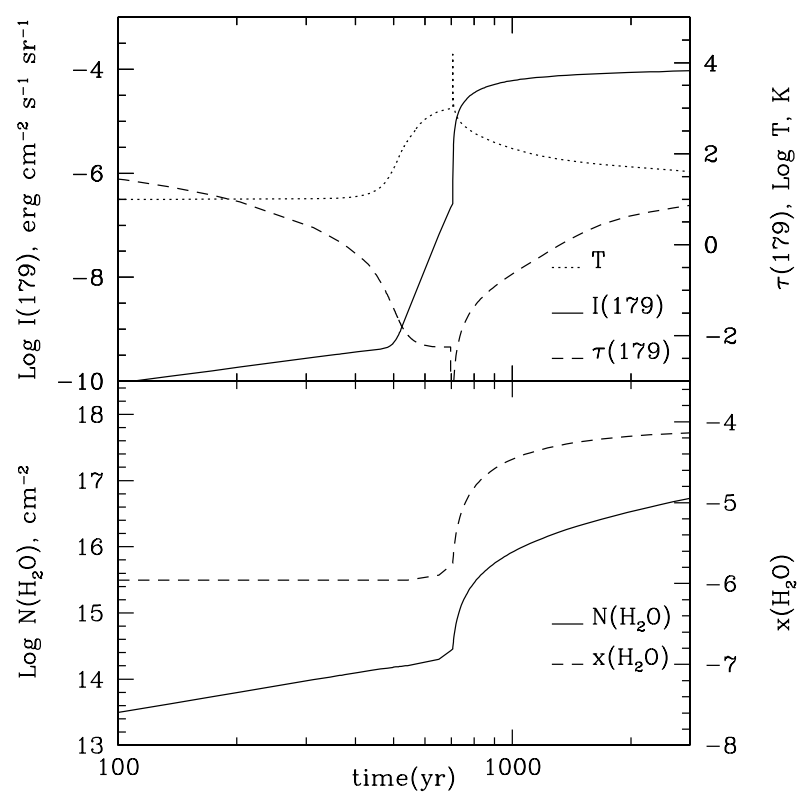

Fig. 10. Top: output of the LVG model (in the framework of the $\mathrm{C}+\mathrm{J}$ model): $179 \mu \mathrm{m}$ line brightness and optical depth. The neutral temperature is also shown for comparison. Bottom: water column density and abundance predicted by the $(\mathrm{C}+\mathrm{J})$ model.

the discrepancy between water abundance measurements and predictions from classical non-dissociative shock models $\left(x\left(\mathrm{H}_{2} \mathrm{O}\right)\right.$ larger than $10^{-4}$, Kaufman \& Neufeld 1996) can be better reconciled in the framework of J-type shock with magnetic precursor models. Moreover, since gaseous water survives only in high-temperature regions, we expect that its emission arises in compact parts of the shock: by normalizing the predicted $179 \mu \mathrm{m}$ line brightness to the observed flux, we derive, even considering the diffraction at the line wavelength, $\theta \approx 30$ arcsec. Therefore, the water emission region is more compact than that emitting the warm $\mathrm{CO}$ by about a factor of four. Noticeably, a difference in the shock area for $\mathrm{CO}$ and $\mathrm{H}_{2} \mathrm{O}$ lines, signals some inconsistencies in 1D geometry, since in this approximation the gradients within the cooling zone (perpendicular to the shock surface) are already taken into account and thus the emitting area should be exactly the same for all the species.
Table 8. Molecular parameters derived for the J-type shock with magnetic precursor model.

\begin{tabular}{cccc}
\hline \hline Species & $\begin{array}{c}N \\
\left(\mathrm{~cm}^{-2}\right)\end{array}$ & $\begin{array}{c}\theta \\
(\operatorname{arcsec})\end{array}$ & $\langle x\rangle$ \\
\hline $\mathrm{H}_{2}$ & $7.4 \times 10^{20}$ & - & - \\
$\mathrm{CO}$ & $1.2 \times 10^{17}$ & 60 & $1.6 \times 10^{-4}$ \\
$\mathrm{H}_{2} \mathrm{O}$ & $5.4 \times 10^{16}$ & 30 & $7.3 \times 10^{-5}$ \\
$\mathrm{OH}$ & $1.3 \times 10^{14}$ & - & $1.8 \times 10^{-7}$ \\
\hline
\end{tabular}

As regards the $\mathrm{OH}$ molecule, we have detected only two lines at 119 and $163 \mu \mathrm{m}$, each connecting the lowest energy levels of the two ladders $\left({ }^{2} \Pi_{3 / 2}\right.$ and $\left.{ }^{2} \Pi_{1 / 2}\right)$ into which the $\mathrm{OH}$ energy level diagram is split. These lines have a similar excitation temperature and critical density, thus not allowing us from discriminating between different shock categories. Using the $\mathrm{C}+\mathrm{J}$ shock structure, the LVG computation predicts a 119/163 ratio of 14 , which is substantially different from the observed value of 2 . This discrepancy could be due to the pumping effect of a dust continuum, the heating of which is not accounted for in our shock model. Indeed, the $163 \mu \mathrm{m}$ line brightness is increased more than that of the $119 \mu \mathrm{m}$ by dust pumping (Offer \& van Dishoeck 1992).

In summary, our multi-species analysis leads us to consider the $(\mathrm{C}+\mathrm{J})$ shock as the model which best reproduces the overall observed molecular emission. The parameters relative to the FIR emission are given in Table 8. Here, we report the molecular column densities and the fractional abundances predicted by the $(\mathrm{C}+\mathrm{J})$ shock model and integrated along the shock profile, along with the angular sizes of the emitting regions derived by the ratio of the predicted brightnesses with the observed fluxes.

\subsubsection{Atomic emission}

Of the atomic and ionic species detected in HH54, oxygen is the only one involved in the same chemical reactions as $\mathrm{H}_{2} \mathrm{O}$ and $\mathrm{OH}$. Therefore, it seems reasonable to expect that a sizeable fraction of the emission at 63 and $145 \mu \mathrm{m}$ should arise in the same shock as the molecular lines. In Table 9 we give the line brightness of the [OI] 63 and $145 \mu \mathrm{m}$ predicted by the three shock models considered in this paper. Even for the highest values (predicted by the $(\mathrm{C}+\mathrm{J})$ model), an emitting area fifty times larger than the ISO beam is required in order to 
Table 9. [O I]63,145 $\mu \mathrm{m}$ brightness as predicted by shock models.

\begin{tabular}{ccc}
\hline \hline Model & $\begin{array}{l}I(63) \\
\left(\mathrm{erg} \mathrm{cm}^{-2} \mathrm{~s}^{-1} \mathrm{sr}^{-1}\right)\end{array}$ \\
\hline $\mathrm{C}$ & $9.9 \times 10^{-7}$ & $9.1 \times 10^{-8}$ \\
$\mathrm{~J}$ & $3.2 \times 10^{-8}$ & $1.5 \times 10^{-9}$ \\
$\mathrm{C}+\mathrm{J}$ & $1.3 \times 10^{-6}$ & $1.4 \times 10^{-7}$ \\
\hline
\end{tabular}

reproduce the observed flux of the $63 \mu \mathrm{m}$ line. This suggests that oxygen emission originates in gas excited by a different mechanism to that which gives rise to the $\mathrm{H}_{2}$ line emission. Both the $F W H M$ of the $63 \mu \mathrm{m}$ line profile and the observed 63/145 ratio of 23 are typical of radiative J-shock environments (e.g. Hollenbach \& McKee 1989), which could form at the apex of the bow-shock or in reverse shocks along the jet. If we compute the mass-flux from the $63 \mu \mathrm{m}$ flux using the formula $\dot{M}_{\text {shock }}=$ $10^{-4} M_{\odot} / \mathrm{yr} \times\left(L(63 \mu \mathrm{m}) / L_{\odot}\right)($ e.g. Liseau et al. 1997) we find $\dot{M}_{\text {shock }}=1.2 \times 10^{-6} M_{\odot} / \mathrm{yr}$, which is similar to the jet mass flux estimated in Sect. 3.2.2.

With regard to the other atomic and ionic species, none of the observed lines can be accounted for by the $(C+J)$ shock model: the predicted intensities of [Fe II], [C I] and [S II] are approximately 4 orders of magnitude smaller than are observed. Indeed, Spitzer maps (Neufeld et al. 2006) have shown that the bulk of the fine structure emission (from [Ne II], [S I], [Si II], [Fe II]) comes from a defined region close to the head of the bow: our results suggest that the degree of dissociation and ionization in this region are much higher than in the gas in which the $\mathrm{H}_{2}$ emission originates. The same authors state that the fine structure lines originate in fast J-type shocks of velocity $\approx 35-$ $90 \mathrm{~km} \mathrm{~s}^{-1}$, in agreement with the (rough) estimate we derived from the [O I] $63 \mu \mathrm{m}$ line for the shock velocity.

In conclusion, the atomic/ionic emission appears to trace a different (faster and more ionizing) shock than the $\mathrm{H}_{2}$ emission. Similar results have already been obtained by us when fitting the near-infrared lines observed in HH99 ( $\mathrm{M}^{\mathrm{c}}$ Coey et al. 2004): in that case, in a self-consistent scenario, two J-shock components, one with a magnetic precursor and one fully dissociative, were required to account for the $\mathrm{H}_{2}$ and the ionic emissions, respectively. However, we postpone a full analysis of this topic to a later paper.

\section{Conclusions}

We have presented a multi-frequency/multi-species analysis of the molecular emission in HH54, by means of one of the most complete spectroscopic data-base in the infrared (from 1 to $200 \mu \mathrm{m})$ ever collected for an $\mathrm{HH}$ object. The $\mathrm{H}_{2}$ lines $(1-12 \mu \mathrm{m})$ coming from levels $v=0$ to $v=4$ with excitation energies between 2000 and $25000 \mathrm{~K}$ have been interpreted in the context of a state-of-art shock code. Two models (a steady-state J-type shock and a quasi-steady J-type shock with magnetic precursor) yield satisfactory fits to the data, allowing us to discard steady-state C-type shocks as a mechanism for the line excitation. The output parameters (temperature and density profiles, molecular species number density and velocity gradient) from these models are adopted as input to an LVG computation to interpret the emission of $\mathrm{CO}, \mathrm{H}_{2} \mathrm{O}$ and $\mathrm{OH}$ in the far-infrared. This second step of the analysis allows us to definitively select the J-type shock with magnetic precursor as the one able both to account for the overall emission of $\mathrm{H}_{2}, \mathrm{CO}$ and $\mathrm{H}_{2} \mathrm{O}$. None of the considered models, however, is able to reproduce the observed $\mathrm{OH}$ emission, which probably could be accounted for by considering the heating of dust grains in the shock model. A further, fully dissociative, high-velocity shock is thought to be responsible for the observed atomic/ionic lines. If it is the case, a picture may emerge in which the optical and near-IR ionic lines trace a fragmented jet shock, the near-IR $\mathrm{H}_{2}$ lines trace ambient J-shocks driven by the jet shocks, and the $v=0 \mathrm{H}_{2}$ lines trace the continuos precursor propagating into the ambient cloud.

Finally, the adopted approach has been extended to provide predictions of the best diagnostic $\mathrm{H}_{2} \mathrm{O}$ line intensities in the range accessible to the forthcoming facilities HIFI and PACS aboard the Herschel satellite.

Acknowledgements. We thank the referee for a detailed and constructive report. S.C. gratefully acknowledges the support of Marc Sauvage with ISOCAM data planning and analysis tools. This work was partially supported by the European Community's Marie Curie Research and Training Network JETSET (Jet Simulations, Experiments and Theory) under contract MRTN-CT-2004-005592.

\section{References}

Bachiller, R. 1996, ARA\&A, 34, 111

Bergin, E. A., Neufeld, D. A., \& Melnick, G. J. 1998, ApJ, 499, 777

Cabrit, S., Bontemps, S., Lagage, P. O., et al. 1999, in The Universe as seen by ISO, ed. P. Cox, \& M. F. Kessler, 449

Caratti o Garatti, A., Giannini, T., Nisini, B., \& Lorenzetti, D. 2006, A\&A, 449, 1077

Cesarsky, C. J., Abergel, A., Agnèse, P., et al. 1996, A\&A, 315, L32

Chernin, L., Masson, C., Gouveia dal Pino, E., \& Benz, W. 1994, ApJ, 426, 204

Chièze, J.-P., Pineau des Forêts, G., \& Flower, D. R. 1998, MNRAS, 295, 672

Chrysostomou, A., Hobson, J., Davis, C. J., Smith, M. D., \& Berndsen, A. 2000, MNRAS, 314, 229

Cuby, J. C., Lidman, C., Johnson, R., Jaunsen, A., \& Moutou, C. 2004, ISAAC User Manual

de Graauw, Th., \& Helmich, F. P. 2001, in The Promise of the Herschel Space Observatory, ed. G. L. Pilbratt, J. Cernicharo, A. M. Heras, T. Prusti, \& R. A. Harris, 45

Draine, B. T. 1980 , ApJ, 241, 102

Downes, T. P., \& Cabrit, S. 2003, A\&A, 403, 135

Dubernet, M.-L., \& Grosjean, A. 2002, A\&A, 390, 793

Flower, D. R. 2001, J. Phys. B, 34, 2731

Flower, D. R., \& Pineau des Forêts, G. 2003, MNRAS, 343, 390

Flower, D. R., Le Bourlot, J., Pineau des Forêts, G., \& Cabrit, S. 2003, MNRAS, 341, 70

Giannini, T., Lorenzetti, D., Tommasi, E., et al. 1999, A\&A, 346, 617

Giannini, T., Nisini, B., \& Lorenzetti, D. 2001, ApJ, 555, 40

Giannini, T., Nisini, B., Caratti o Garatti, A., \& Lorenzetti, D. 2002, ApJ, 570, L33

Giannini, T., MCoey, C., Caratti o Garatti, A., et al. 2004, A\&A, 419, 999

Graham, J. A., \& Hartigan. P. 1988, ApJ, 95, 4

Gredel, R. 1994, A\&A, 292, 580 (G94)

Green, S., Maluendes, S., \& McLean, A. D. 1993, ApJ, 85, 181

Grosjean, A., Dubernet, M.-L., \& Ceccarelli, C. 2003, A\&A, 408, 1197

Hartigan, P., Raymond, J., \& Hartmann, L. 1987, ApJ, 316, 323

Hartigan, P., Morse, J. A., \& Raymond, J. 1994, ApJ, 436, 125

Hollenbach, D., \& McKee, C. F. 1989, ApJ, 342, 306

Hughes, J., \& Hartigan, P. 1992, AJ, 104, 680

Kaufman, M. J., \& Neufeld, D. A. 1996, ApJ, 456, 611

Knee, L. B. G. 1992, A\&A, 259, 283 (K92)

Le Bourlot, J., Pineau des Forêts, G., Flower, D. R., \& Cabrit, S. 2002, MNRAS, 332, 985

Lesaffre, P., Chièze, J.-P., Cabrit, S., \& Pineau des Forêts, G. 2004a, A\&A, 427, 157

Lesaffre, P., Chièze, J.-P., Cabrit, S., \& Pineau des Forêts, G. 2004b, A\&A, 427, 147

Lidman, C., Cuby, J.-C., \& Vanzi, L. 2002, SofI User Manual

Liseau, R., Ceccarelli, C., Larsson, B., et al. 1996, A\&A, 315, L181

Liseau, R., Giannini, T., Nisini, B., et al. 1997, in Herbig-Haro Flows and the Birth of Low Mass Stars, ed. B. Reipurth, \& C. Bertout, 111

Mc Coey, C., Giannini, T., Flower, D. R., \& Caratti o Garatti, A. 2004, MNRAS, 353,813

McKee, C. F., Storey, J. W. V., Watson, D. M., \& Green, S. 1982, ApJ, 259, 647

Neufeld, D. A., Melnick, G. J., \& Harwit, M. 1998, ApJ, 506, L75 
Neufeld, D. A., Melnick, G. J., Sonnentrucker, P., et al. 2006, ApJ, in press [arXiv: astro-ph/0606232]

Nisini, B., Lorenzetti, D., Cohen, M., et al. 1996, A\&A, 315, L321

Nisini, B., Benedettini, M., Giannini, T., et al. 1999, A\&A, 350, 529

Offer, A. R., \& van Dishoeck, E. F. 1992, MNRAS, 257, 377

Offer, A. R., van Hernert, M. C., \& van Dishoeck, E. F. 1994, J. Chem. Phys., 100,362

Ott, S., Gastaud, R., Ali, B., et al. 2001, in ASP Conf. Ser. 238, Astronomical Data Analysis Software and Systems X, ed. F. R. Harnden, Jr., F. A. Primini, \& H. E. Payne (San Francisco: ASP)

Phillips, T. R., Maluendes, S., \& Green, S. 1996, ApJSS, 107, 467

Poglitsch, A., Waelkens, C., \& Geis, N. 2001, in The Promise of the Herschel Space Observatory, ed. G. L. Pilbratt, J. Cernicharo, A. M. Heras, T. Prusti, \& R. A. Harris, 29

Reipurth, B., \& Bally, J. 2001, ARA\&A, 39, 40

Rieke, G. H., \& Lebofsky, M. J. 1985, ApJ, 288, 618
Rousselot, P., Lidman, C., Cuby, J.-G., Moreels, G., \& Monnet, G. 2000, A\&A, 354,1134

Sandell, G., Zealey, W. J., Williams, P. M., Taylor, K. N. R., \& Storey, J. V. 1987, A\&A, 182, 237

Schöier, F. L., van der Tak, F. F. S., van Dishoeck, E. F., \& Black, J. H. 2005, A\&A, 432, 369

Schwartz, R. D. 1977, ApJS, 35, 161

Schwartz, R. D., \& Dopita, M. A. 1980, ApJ, 236, 543

Smith, M. D., \& Mac Low, M.-M. 1997, A\&A, 326, 801

Smith, M. D., \& Rosen, A. 2003, MNRAS, 339, 133

Smith, M. D., Froebrich, D., \& Eislöffel, J. 2003, ApJ, 592, 245

Swinyard, B. M., Clegg, P. E., Ade, P. A. R., et al. 1996, A\&A, 315, L438

Thai-Q-Tung, Dinh-V-Trung, Nguyen-Q-Rieu, et al. 1998, A\&A, 331, 317

Wilgenbus, D., Cabrit, S., Pineau des Forêts, G., \& Flower, D. R. 2000, A\&A, 356,1010 